\title{
Predictability of the antigenic evolution of human influenza A H3 viruses
}

\author{
Authors: R. Aguas ${ }^{1}$, N.M. Ferguson ${ }^{1}$
}

\section{Affiliations:}

$5{ }^{1}$ MRC Centre for Outbreak Analysis and Modelling, Department of Infectious Disease Epidemiology, Imperial

6 College London, Faculty of Medicine, Norfolk Place, London, UK. 


\section{Abstract}

8 The current influenza A antigenic evolution paradigm suggesting that antigenic evolution is highly constrained, with

9 successful new viruses being near optimal at maximizing their antigenic distance from past strains. This begs the

10 question of whether influenza's antigenic evolution is fundamentally predictable, or if it takes place on a much

11 higher dimensional antigenic space with multiple possible trajectories. We tackle this issue by building a genotype

12 to phenotype map validated on historical hemagglutination inhibition assay data by using machine learning methods.

13 This map uses amino acid physiochemical properties for inference, learning the expected antigenic distance given

14 the differences in polarity and hydrophobicity observed across any two viral sequences, and is thus applicable to

15 newly sampled viruses with previously unseen amino acids. This allows us to accurately blindly predict the

16 antigenic relevance of soon to be vaccine viral strains. We couple the genotype to phenotype map with a molecular

17 evolutionary simulation algorithm to explore the limits of influenza's antigenic evolution and infer to what extent it

18 is in fact predictable. Although we do uncover some canalization of antigenic trajectories, we find that multiple

19 antigenic lineages are equally viable at any one point in time even though typically only one of those trajectories is

20 actually realized. 


\section{Introduction}

Seasonal influenza epidemics remain a major public health concern, resulting in 250,000 to 500,000 deaths per year [1]. Human influenza A evolution is characterized by rapid evolution yet limited extant genetic diversity at any point in time - resulting in a characteristic single trunk phylogeny $[2,3]$. At the molecular level, antigenic drift is mainly determined by mutations in two viral segments encoding for surface proteins hemagglutinin (HA) and neuraminidase (NA), which are in direct contact with the host's immune system [4,5]. Hemagglutination inhibition (HI) assays provide an empirical representation of antigenic drift by measuring differential antibody neutralizing responses across a panel of viruses. Antigenic maps built from HI data reveal a punctuated evolutionary pattern, with periods of relative stasis (defining antigenic clusters) followed by selective sweeps by antigenically novel strains (cluster jumps)[3]. The linearity of these antigenic maps suggests that antigenic evolution is highly constrained, with successful new viruses being near optimal at maximizing their antigenic distance from past strains. One crucial standing question is then the extent to which antigenic evolution is fundamentally predictable, or if antigenic evolution takes place on a much higher dimensional antigenic space with multiple possible trajectories. Here we address these questions using machine learning methods to predict antigenic distances from differences in physiochemical properties in influenza HA1 sequences. The genotype to phenotype (GP) map thus formulated for influenza antigenicity can reproduce the observed features of the antigenic evolution of influenza $\mathrm{A} / \mathrm{H} 3 \mathrm{~N} 2$ viruses and accurately predicts the antigenic novelty of viruses one year ahead. By coupling our GP map with a molecular evolution algorithm, we unveil how HA1 as a whole has evolved near neutrally whilst epitope sites have undergone strong positive selection and give insights into the predictability of flu antigenic evolution. Our results suggest that 
influenza's observed evolutionary pathway is but one of many plausible trajectories, with mutations in antigenically relevant sites generating a breadth of antigenically novel viruses later subject to epidemiological selection pressures that limit the observed viral diversity.

Modelling studies have generated competing hypotheses about the key evolutionary and epidemiological determinants of observed influenza A evolutionary patterns [6-9], but definitive understanding is lacking. Influenza A phylodynamics have been simulated assuming comparably parsimonious, yet fundamentally different mechanistic processes: short-term subtype transcending immunity [6]; positive selection associated with cluster transitions in an otherwise neutrally evolving network [7]; reuse of a limited number of antigenic combinations [8]; accumulation of deleterious mutations genetically linked to beneficial ones. More recently, a more phenomenological model has successfully reproduced observed trends in influenza evolution and epidemiology [10].

One important question where existing models differ is the extent to which antigenic evolution is fundamentally predictable $[10,11]$ - or even deterministic [10] - or if the space of possible trajectories is much higher dimensional, with but one realized trajectory (e.g. due to stochasticity [7] or pruning of alternative lineages [6]). In a more narrow sense, recent studies have put forward algorithmic predictions of which genetic variants of an emerging viral set [12,13] will come to dominate circulating viruses in the next year or two (and thus be suitable vaccine candidate strains). However, predicting the antigenic properties of previously unseen strains multiple years ahead remains an open challenge. Whether or not it's possible to predict de novo which strains will emerge in the future ahead of their identification at even low frequency is 
completely unclear; the answer fundamentally depends on the extent to which influenza evolution is deterministic.

Here we address these questions using machine learning methods to predict the expected antigenic distance between two influenza $\mathrm{A} / \mathrm{H} 3 \mathrm{~N} 2$ viruses given the differences in physiochemical properties across their respective HA1 sequences. We focused on HA1 since it appears particularly relevant for viral antigenic evolution, being the principal target of antibody mediated immunity $[14,15]$ and containing several empirically established antigenic sites $[16,17]$.

\section{Results}

\section{Genotype to Phenotype Map}

The HI assay outputs a viral titer which indicates the number of times the sera needed to be diluted for hemagglutination of the antigen and red blood cells to occur [18]. This suggests that there are electrostatic interactions between antigen and antibodies which are disrupted when the serum is too diluted. We thus postulated that physiochemical property differences between the amino acid sequences of a given antigen/serum pair can serve as a proxy for the antigenic distances observed in HI assays, thus formalizing a genotype-to-phenotype (GP) map. To do so, we implemented a machine learning algorithm that was informed on how differences in 54 distinct physiochemical properties relate to empirical HI titre measurements - more detailed description of this method can be found in S1 Text). Out of those 54 properties, a subset of 7 ( $\alpha$ helix, $\beta$ sheet, $\beta$ turn, bulkiness, coil, polarity and hydrophobicity) emerged as the most relevant in explaining the observed phenotype, with polarity and hydrophobicity being the most significant. S1 Figure clearly shows that differences in hydrophobicity and polarity found in 
HA1 have a very high degree of concordance with genetic distances across influenza H3 viral

91 sequences collected over time as well as historic antigenic maps [3]. By using vectors of amino

92 acid hydrophobicity and polarity rather than the actual amino acid sequences, we allow the

93 resulting GP map to be used to predict the antigenic characteristics of novel influenza strains

94 with previously unseen amino acid polymorphisms. We constructed the GP map using historic

95 HI data [3] and corresponding HA1 sequences (S1 Table), by training a Random Forest

96 Algorithm (RFA) to learn the expected antigenic distances between two viruses (measured in

97 integer 2-fold dilutions given by the HI assays) given the differences in amino acid polarity and

98 hydrophobicity across their respective viral sequences. The resulting classifier is able to predict

99 the measured antigenic distances exactly for $\sim 90 \%$ of the antigen/serum pairs (Figure 1A) and

100 successfully reproduces the antigenic map of the H3N2 subtype (Figure 1B). 
a

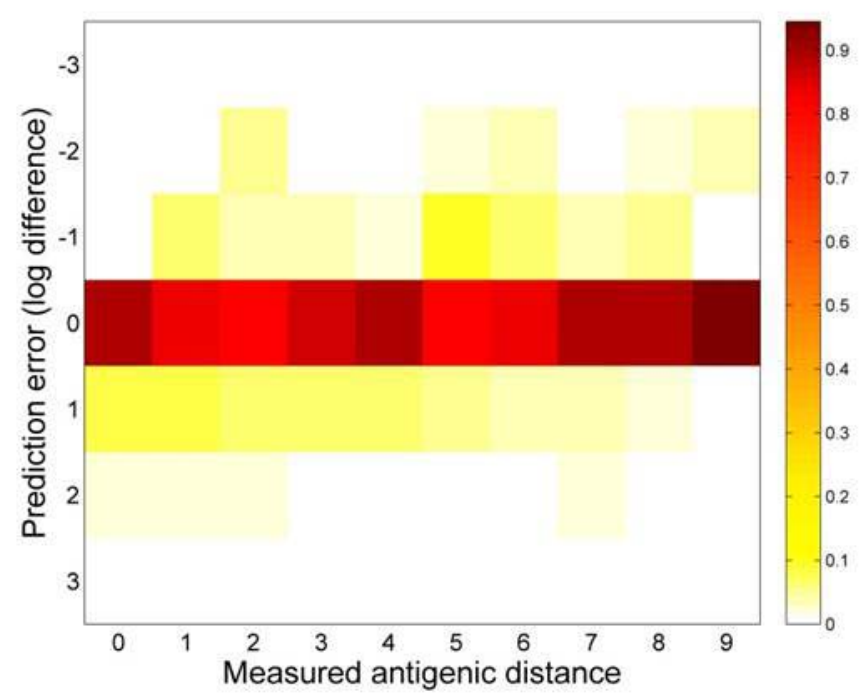

b

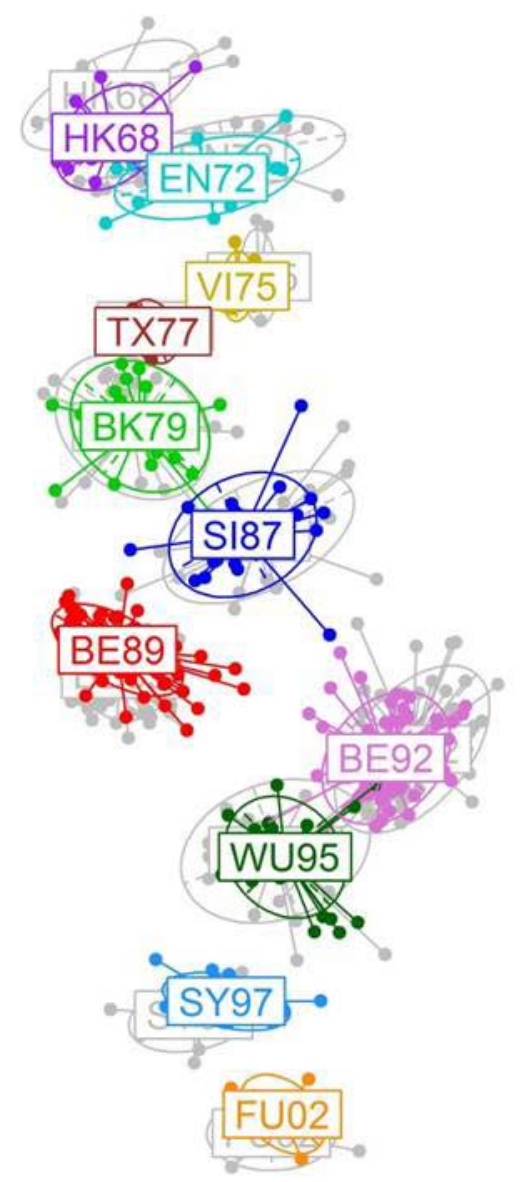

101 
distance (distance measured on a $\log -2$ scale) for every antigen-serum pair. Antigenserum pairs are grouped by measured antigenic distance on the horizontal axis. The colour of each rectangular pixel illustrates the distribution of predictive errors for pairs with a particular measured antigenic. A measure of $90 \%$ for prediction error 0 indicates that $90 \%$ of antigen/serum pairs antigenic distances are predicted exactly. Superimposed 2-dimensional antigenic maps generated from the RFA predicted antigenic distances (in colour) and from the HI titre data (in grey). See Methods for details of the maximum-likelihood algorithm used to construct these maps.

\section{Predicting the antigenic novelty of previously unseen viruses}

While Figure 1 demonstrates the ability of the GP map to reproduce the data on which it was trained, this approach is also able to predict the antigenic characteristics of strains not used to train the RFA. To determine whether newly sampled viruses are antigenically novel, we train the RFA up to a specific point in time and predict the antigenic distance between all antigen/serum pairs constituted by an element of the current antigenic cluster and another element collected in the year following the reference time point. Mean one year ahead antigenic distances predicted for each year of collection show a remarkable agreement with the measured antigenic distances, particularly highlighting the antigenic novelty of future vaccine strains - Figure 2. These mean values are a compound of predicted distances to future members of the current antigenic cluster and to members of new yet unobserved clusters, since some viruses collected in cluster transition years such as 2002 e.g., can still be members of the currently circulating cluster at the time of prediction (SY97 in this example) - S2 Table. 


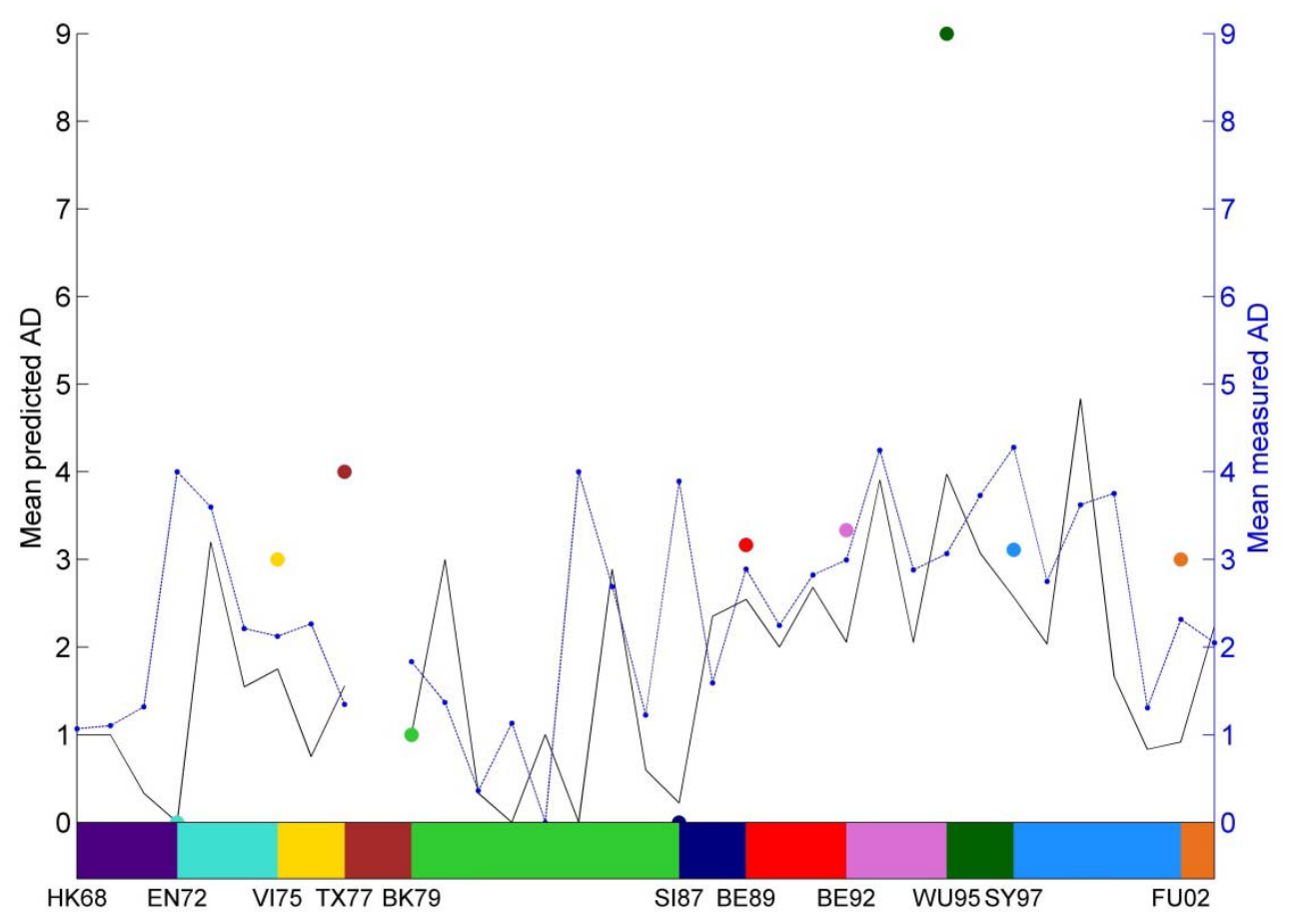

Figure 2. Predicting the antigenic novelty of newly sequenced viruses one year ahead. The black line shows the mean predicted antigenic distance between antigens collected in each year and sera collected in the three previous years, given an RFA trained on HI data containing antigen/serum pairs sampled up to the previous year. The colored dots identify the mean predicted antigenic distances referring to antigens which turned out to be vaccine strains, whereas the blue line represents the mean empirical antigenic distances observed for all antigen/serum pairs used for prediction each year. The different colors map vaccine strains onto their antigenic cluster, longitudinally represented as colored blocks.

S2 Figure further illustrates the RFA prediction errors resulting from out-of-sample predictions, particularly across clusters. Given a GP map trained up to data collected in 1995, predicted 
antigenic distances for viruses appearing one year into the future are very similar to the measured distances (>75\% having an error of $\pm 1 \log -2$ unit of antigenic distance or less), as might be expected given antigenic variation is quite limited within the same antigenic cluster (for reference, the mean antigenic distance between viruses in adjacent clusters is $4.46( \pm 1.3))$. The following year saw a cluster transition (WU95 $\rightarrow$ SY97), which reduced predictive accuracy somewhat since cluster transitions are typically associated with the fixation of previously unrecorded alleles [11,19], meaning the RFA is potentially blinded to the significance of mutations in those amino acids. Hence, while just under $70 \%$ of predicted distances are within 1 $\log -2$ unit of the observed antigenic distance when looking 2 to 8 years into the future, a small proportion of observations have higher prediction errors associated with them, and this proportion increases slowly the further ahead one tries to predict. However, our GP map can predict the qualitative pattern of antigenic evolution even across two antigenic cluster jumps (S2C Figure).

\section{Influenza's molecular evolution and selection for differences in physiochemical properties}

The biological plausibility of our approach can be investigated by comparing the amino-acid positions in HA1 which the RFA identifies as significant in predicting antigenicity, with those of known epitopes (Figure 3). We find that the set of significant positions identified by the RFA as antigenically relevant, maps closely onto HA1 sites with the highest ratio of non-synonymous to synonymous mutations, with most lying in epitopes (the 25 amino acid positions with the highest significance are listed in S3 Table), providing an improved view of the physio-chemical constraints at play in influenza antigenic evolution [20]. Furthermore, we uncover a significant amount of antigenically relevant interactions in H3 hemagglutinin, as suggested previously 
$163[21,22]$. We show that the importance of many amino acid positions depends on the time frame

164 of the training set (Figure 3), with some locations being significant throughout. The predicted

165 RFA significance for each amino acid is quite closely related to the total number of non-

166 synonymous mutations observed at those positions, reinforcing the suggestion that positive

167 selection is operating on a relatively small subset of HA1 amino acids - S3 Figure. This apparent

168 association of accumulation of non-synonymous mutations with increased phenotypic plasticity

169 deserves further consideration. Examination of the genetic evolutionary trajectories of epitopic

170 and non-epitopic sites in HA1 reveals that if HA1 amino acids are mutating exclusively under

171 neutrality assumptions (using a Kimura model with 2:1 transition to transversion ratio and equal

172 mutation probability for each position), the simulated viruses generally increase their differences

173 in polarity and hydrophobicity when compared to their ancestral HK69 strain in a liner fashion.

174 Framing those neutral expectations with the observed trends, we see that while HA1 as a whole

175 has evolved near neutrally (S4A Figure), epitopes have undergone strong positive selection,

176 optimizing their polarity and hydrophobicity differences relative to the ancestral HK68 virus

177 (S4B Figure). 


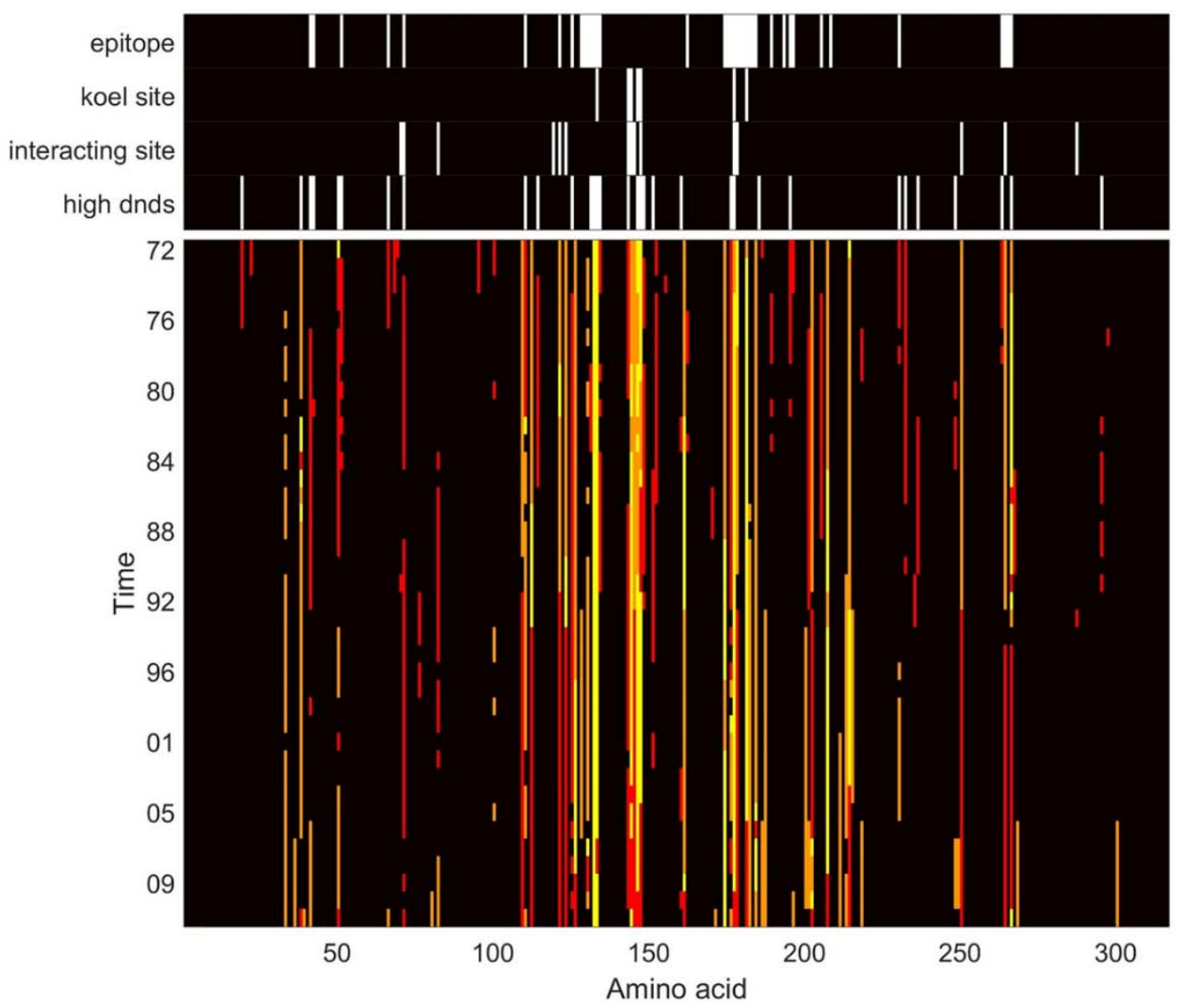
antigenic distances between antigen/sera pairs of sequences. RFAs were performed using training data encompassing different time spans. If a particular HA1 position was significant in an RFA trained on data collected from a specific time point onwards, that position is colored orange. If that position is significant in the RFA trained on data from 1968 up to that time point, the position is colored red. If the position is significant in both of those time frames, it is shown in yellow. The significant positions in the RFA map closely to positions with a high ratio of non-synonymous to synonymous mutations, with most occurring on known HA1 epitopes (highlighted in white above). 
It seems then that there are a few key amino acids in HA1 displaying a larger than expected phenotypic plasticity, evident in the range of observed polarity and hydrophobicity differences as well (S5 Figure), which seem to be under positive selection. A detailed analysis of how mutations are distributed across HA1 reveals a surprisingly stable pattern, with approximately $50 \%$ of all mutations across any antigen/serum pair falling on this subset of highly phenotypically relevant sites identified by the RFA - S6 Figure. Other epitopes are underrepresented, whilst about a third of mutations lies on non-epitopic polymorphic sites.

\section{Exploring evolutionary trajectories and the limits of antigenic evolution predictability}

Having demonstrated that it is possible to predict the antigenic characteristics of new H3N2 strains several years into the future with a good but not perfect level of accuracy (Figures 2 and S2), we now consider whether it is possible to predict which strains will arise in the future. The linearity of the antigenic map suggests the existence of a strong immune-mediated selection compelling novel influenza viruses to maximize their antigenic distance to previously circulating viruses $[3,10]$. Above, we show how this is manifested as an uneven distribution of nonsynonymous distributions across HA1, resulting in accumulation of differences in physiochemical properties at key sites. Furthermore, a look at how genetic and antigenic evolutionary trajectories match, indicates that both antigenic and genetic differences are much smaller within members of the same cluster than between members of adjacent clusters (S7 Figure). This suggests there is a limited number of genetic evolutionary trajectories which translate into cluster jumps, but does not help resolve whether the observed antigenic evolutionary trends are a result of:

- $\quad$ structural constraints limiting the physio-chemical plasticity of new viruses ("plasticity"). 
- immunological selection pressure, forcing new viruses to accrue physio-chemical differences relative to circulating ones ("antigenic filtering").

- seeding effects, with apparent "canalization" of antigenic and genetic evolutionary trajectories resulting from the expansion of a single viral lineage in a susceptible population ("seeding").

- $\quad$ All the above.

Here, we consider how such constraints shape the joint genetic/antigenic evolution of H3 and how they translate into the possible evolutionary trajectories the virus might follow. We try to investigate these effects independently to address their significance in recovering the observed antigenic maps, specifically trying to reproduce cluster jumps. To do so we use a genetic simulator algorithm coupled with a GP map and compare the predicted antigenic distances between simulated viruses and both the ancestral strain and sampled viruses belonging to the following antigenic cluster. In our genetic simulator we explore 3 molecular evolutionary models, expressing quite different mutation distribution profiles: a) 7-site model, in which mutations are restricted to the sites identified as cluster determinants by Koel and colleagues [23]; b) RFA 25-site model, where mutations are restricted to the 25 most significant amino acid positions as predicted by the random forest trained on the full dataset; c) Generalized model where mutations are distributed according to probabilities directly derived from S6 Figure.

Whilst the latter model is in agreement with the 'canalized' antigenic evolution [10] and the antigenic recycling [8] hypotheses, since the majority of mutations is directed at a small subset of positions, it also allows for new, previously antigenically irrelevant, alleles to become significant [19] and epistatic interactions between antigenically significant sites and other loci [24]. 
234 We first compare the accuracy of the GP map obtained when including all sites in the training set

235 with that of the antigenic maps resulting from antigenic distances predicted by RFAs trained

236 exclusively on the two limited sets of sites listed above. The 25-site RFA model performed

237 comparably to the generalized model in reproducing the training set and in terms of predictive

238 accuracy unlike the 7-site model that, while broadly capturing cluster transitions, was

239 substantially poorer at reproducing the precise size and shape of antigenic clusters (S8 Figure).

240 Thus, while 7-site may be sufficient to explain much about cluster transitions, our analysis

241 suggests that more sites contribute to the fine detail of antigenic variation within clusters, and

242 consequently, finer level antigenic novelty leading to antigenic cluster transitions.

243 To ascertain the validity of our joint GP map and molecular sequence generating tool, we

244 simulated thousands of viruses from a single SY97 cluster ancestor using the generalized

245 evolutionary model and attempt to replicate the antigenic location of the FU02 cluster. Given

246 perfect information as to what positions mutate in the future and using GP map trained on the

247 complete dataset we can almost exactly retrieve the observed antigenic map (S9 Figure). Whilst

248 still intercepting the antigenic space occupied by the empirical future cluster, blinded predictions

249 reflect a degree of uncertainty as to the antigenic trajectory undertaken by flu viruses, with a

250 range of antigenically distinct viruses equally likely to emerge. Indeed, less than $1 \%$ of the

251 simulated viruses were within 2 units of antigenic distance of the empirically realized FU02

252 cluster. Looking deeper into the genetic relationship between simulated sequences and historic

253 ones, we can see that only a minority of the antigenically plausible sequences are genetically

254 very similar to the observed FU02 antigenic cluster (S10 Figure).

255 It then seems that "antigenic filtering" alone cannot reconcile genetic and antigenic H3 256 evolution, so we next examine what roles "plasticity" and "seeding" might have. To do so, we 
compare how the expected mean antigenic distance of simulated $n$-point mutants to their ancestral strain, and between themselves fits with those observed in the empirical data. We introduce "plasticity", "antigenic filtering", and "seeding" effects sequentially, thus always

260 limiting the physio-chemical plasticity of each HA1 site and stacking the other effects in

261 succession. We start with a strain in the SY97 cluster as the ancestor (see S6 Figure for another 262 starting point) and train the RFA only on strains from SY97 and earlier clusters. Figure 4A 263 illustrates how the predicted antigenic distance between simulated viruses with a specific number 264 of mutations to the ancestral strain compares with the predicted antigenic distance between pairs 265 of equally evolved simulated viruses (with same number of mutation to the ancestral). The mean 266 antigenic distance between simulated viruses is consistently larger than their respective antigenic 267 distances to the common ancestor if no "antigenic filtering" is imposed, i.e. in the absence of 268 immune pressure. When antigenic selection is introduced (Figure 4B), the simulated viruses 269 somewhat converge antigenically, being, on average, more similar to each other than to viruses 270 in the ancestral cluster. This effect is more a consequence of the forced increase in the mean 271 antigenic distance between simulated viruses and their ancestor resulting from the immune 272 selection filter (S11 Figure) than a true antigenic convergence, since there isn't a significant 273 decrease in the pairwise antigenic differences between simulated viruses. This would suggest 274 that selection for antigenic novelty could result in any one of many antigenically distinct viral 275 lineages. Whilst these results are clear for both the generalized and 25-site models, they are 276 much less so for the 7-site model (S11 Figure) due its antigenic sensitivity to changes in a very 277 small number of sites. Although mutations can occur in up to 25 amino acids in the later model, 278 only changes in 7 of those actually result in an antigenic effect which then tends to be quite 

the effect of immune selection difficult with this model.

a

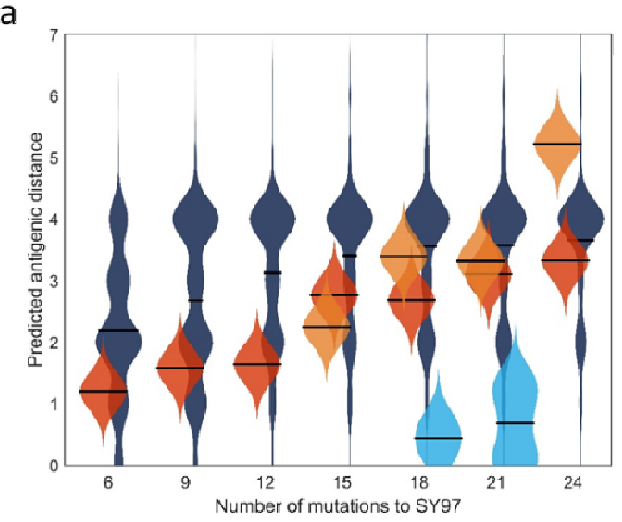

b

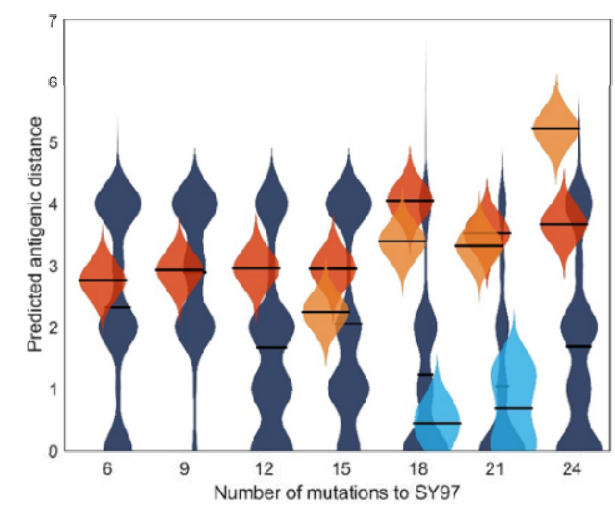

C

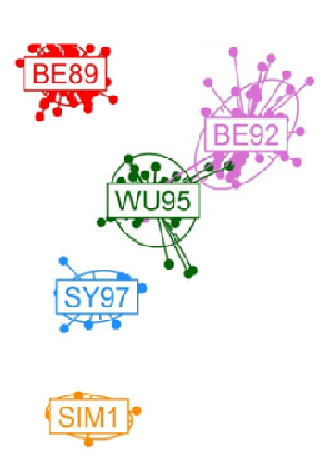

d

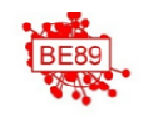

e

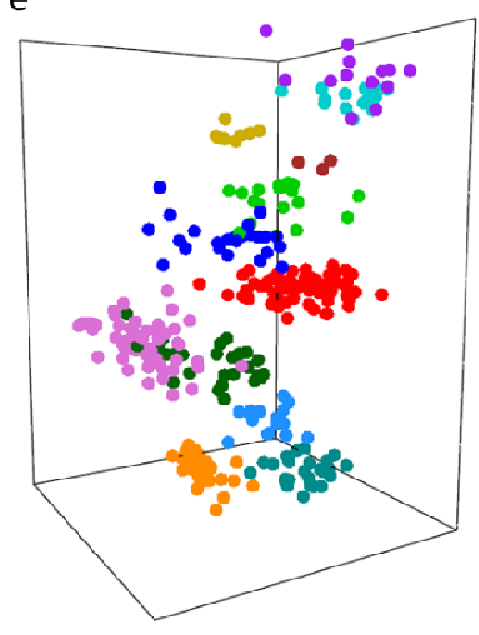

281

Figure 4. Unfiltered (A) and filtered step-wise (B) antigenic evolution from a given ancestral strain. The violin plots illustrate the kernel density estimates for two predicted antigenic distances (horizontal lines represent the mean) on sets of simulated viruses with $\mathrm{X}$ amino acid mutation relative to an ancestral strain (a member of the SY97 cluster). The dark blue violin plots represent the distribution of antigenic distances (with the respective means as horizontal lines) between simulated viruses with $\mathrm{X}$ amino acid mutations relative to a virus in SY97. The red violin plots give the predicted antigenic distances across simulated viruses. Light blue violin plots give the empirical antigenic distances between viruses in SY97 and FU02, whilst the orange plots refer to the measured antigenic distances within the FU02 cluster. (C) Antigenic map of one simulated 

antigenic cluster arising from SY97 (see S1 Text for description of simulation methods). (D, E) Antigenic maps including two simulated clusters both 3-5 antigenic distance units away from their SY97 ancestor, on different spatial embeddings. The 2-Dimensional embedding is unable to properly resolve the antigenic distances between the simulated clusters in contrast with the 3-Dimensional representations (E).

When we simulate a gradual evolutionary process akin to what has been proposed previously [25-27], with multiple related lineages co-circulating globally with occasional expansion of one of those lineages in temperate locations following migration, here termed 'seeding', we observed much stronger antigenic convergence. Quite strikingly, the mean predicted antigenic distance between simulated viruses only matches the observed mean difference among FU02 cluster samples when this stepwise process was implemented (Figures 4B, S8).

\section{Predicting cluster jumps and implications for vaccine updates}

If 'seeding' is indeed a critical process, we need to better characterize which viruses can serve as seeds to a cluster jump. We found that any one of the intermediate simulated viruses resulting from the second mutational step could seed an antigenic cluster which would look nearly identical to the FU02 cluster on an antigenic map (Figure 4C). This is not an indication of the similarity of this simulated cluster with the FU02 cluster but rather a reflection of how the geometry of antigenic maps is mainly determined by large antigenic distances between observed viruses and their antisera. The similar antigenic map location of the observed FU02 cluster and several simulated clusters (we only show a representative one) is solely a result of those clusters sharing the same distance to their ancestor. If two new lineages were to emerge simultaneously, the resulting map would show substantial antigenic separation between them (Figure 4D). We 
315 find that the accuracy of the 2-dimensional map when reproducing pairwise distances between

316 antigen and sera substantially worsens when mapping multiple simulated antigenically divergent

317 clusters of viruses, with a 3-dimensional representation being preferred (Figure 4E). The

318 adequacy of a 2-dimensional representation thus results from the single lineage seen in H3N2

319 HA evolution, rather than necessarily being the fundamental underlying dimensionality of the

320 antigenic shape space which influenza evolution explores [28] - S12 Figure.

321 We show that we can reproduce the observed antigenic patterns of relatedness among simulated

322 viruses and between simulated and sampled viruses, as well as identify viruses that are

323 antigenically novel and likely seeds of new clusters. Next, we push the boundaries of

324 evolutionary predictability by trying to prospectively replicate observed cluster jumps. To do so,

325 we take a late sample (collected the year prior to the establishment of a new cluster) of a given

326 antigenic cluster and simulate viruses according to the generalized model, including all the

327 evolutionary constraints listed above ('plasticity', 'filtering', and 'seeding'). The GP map uses a

328 RFA which is trained on data only up to the year of collection of the strain used as a seed, but

329 here we also calculate distances to sampled viruses in the future cluster, thus comparing how

330 predicted clusters fit with the realized antigenic trajectory. 


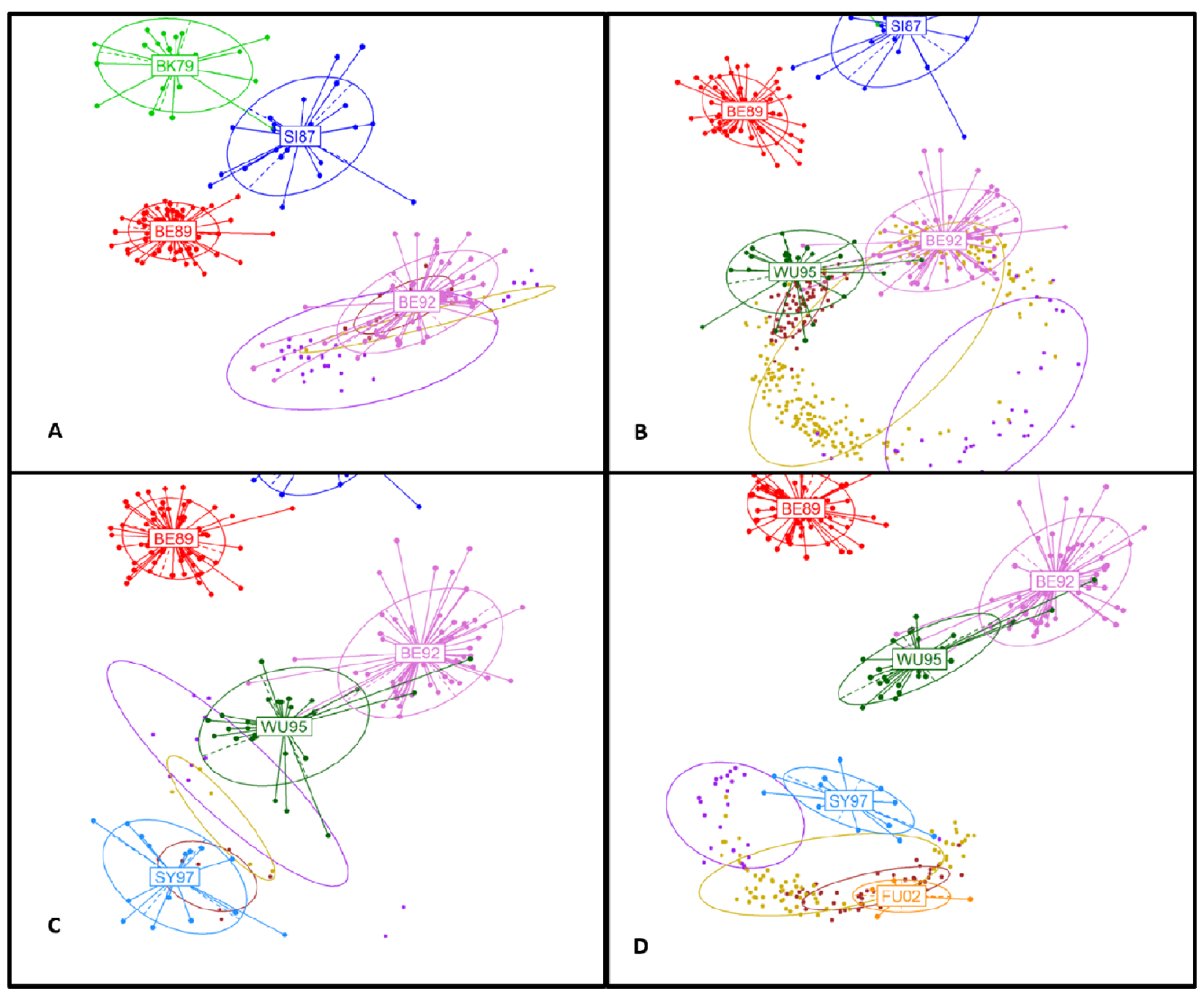

Figure 5. Evolutionary trajectories in antigenic space. The panels illustrate 4 cluster jump predictions. From a given cluster an RFA is trained on data up to a specific date when the chosen ancestral strain was collected, and 100,000 viruses are genetically evolved according to the generalized model. The simulated viruses are classified into distinct groups according to their respective predicted antigenic distances to the observed future cluster and all preceding clusters. The simulated viral groups (or antigenic lineages) are colored purple, yellow and brown in descending order of antigenic similarity to the observed future cluster, respectively. 
341 Strikingly, all simulated cluster jumps reveal the same patterns, with 3 quite distinct antigenic

342 trajectories being theoretically possible, out of which only one resembles the observed cluster

343 (Figure 5). We can identify specific mutations generating pitchforks in the antigenic trajectory

344 followed by simulated viruses - see S1 text for more details. From the set of 6-14 positions

345 highlighted as significant to explain antigenic lineage segregation, there is consistently at least

346 one that has previously been identified (19) as a cluster jump determining mutation - S13 Figure.

\section{Discussion}

348 Here, we have shown how machine-learning based feature selection methods can be used to 349 generate an explicit genotype to phenotype (GP) map that can reproduce the observed features of A/H3N2 influenza antigenic evolution and offers insights into its predictability. Our results

351 indicate that the antigenic distance of newly isolated viruses from their ancestors can be accurately predicted from their HA1 sequence with the methods presented here. This suggests that such GP maps could usefully supplement experimental antigenic characterization of newly sampled viruses in routine virological surveillance for vaccine strain selection. evolution is fundamentally predictable with a narrow set of possible antigenic trajectories the virus can undertake at a point in time. In contrast, our analysis suggests that influenza's observed evolutionary pathway is but one of a limited set of antigenic trajectories, with mutations in antigenically relevant sites (particularly HA1 epitopes, but not exclusively) generating a breadth of antigenically novel viruses later subject to epidemiological selection pressures which limit

361 overall viral diversity [6,10,27]. We conclude that selection for immune escape does not tightly 362 constrain influenza's antigenic evolutionary trajectory but rather that epidemiological selection 363 (with marked winter epidemics and summer bottlenecks) coupled with strong seeding effects 
364 (largely determined by the existing intense global mobility network) determine the observed antigenic trajectory. For all the counterfactual antigenic trajectories to be observed, a much more intense and constant sampling effort would be required.

367 Clearly there are limitations to the use of GP maps, in that they can only be trained on what has been observed. While we partially mitigated this limitation by training our RFA model on changes in physiochemical properties of HA amino-acids rather than the amino-acid sequences directly, mechanistic models predicting the conformation and antigenicity of HA from its sequence would clearly be preferable. However, these remain a long-term goal. Our work indicates that machine-learning derived GP maps, coupled with judicious animal studies [23], are now able to give substantial scientific insight into the phenotypic evolution of influenza viruses as well of being of practical benefit in enhancing virological surveillance.

\section{Materials and Methods}

377 Genotype-to-phenotype (GP) map: We trained a Random Forest Algorithm (RFA) on a dataset of pairwise differences in physiochemical properties between the amino acid sequences of antigen/serum pairs and the respective antigenic distances measured by the HI assay. The RFA is an ensemble classifier consisting of multiple low correlation decision trees, which aggregate into

381 a low bias and low variance "forest". Each tree in a random forest is trained on a random subset

382 of the data, and each tree branch contains randomly chosen variables from all available variables

383 (in this case, a subset of positions in the amino acid sequences for each branch). Final 384 classification of each sample results from aggregating the votes of all trees in the forest, with the 385 importance measure for each variable being the loss of classification accuracy caused by the 386 random permutation of attribute values for that variable. Overall, RFA displays excellent 
387 performance in classification tasks [30,31] and provides direct measures of both variable

388 importance and classification error. We used the RandomForest $\mathrm{R}$ package to implement the

389 RFA. For more information see S1 Text.

390 Antigenic mapping: Antigenic mapping [3,28], by offering a simple intuitive representation of 391 large volumes of $\mathrm{HI}$ assay data, is a useful tool for unveiling the antigenic evolution of a 392 pathogen. It is fundamentally a multidimensional scaling (MDS), and more specifically, a metric unfolding problem (due to the sparseness of the data). Assuming the empirical HI data is directly related to Euclidian distances in an antigenic space, we can view the problem of map creation as that of the minimization of an error function, $E$ :

$396 \quad E=\sum_{i, j=1}^{i, j=M, N}\left(D_{i j}^{\text {data }}-D_{i j}^{\text {map }}\right)^{2} \quad ; \quad D_{i j}^{\text {map }}=\left|X_{i}-X_{j}\right|^{2}$

397 where $D_{i j}^{\text {data }}$ correspond to the measured antigenic distances, and $D_{i j}^{\text {map }}$ to the Euclidean distances computed between the location, $X_{i}$, of the $i^{\text {th }}$ of $M$ antigens in shape space, and $X_{j}$, the location of the $j^{\text {th }}$ of $N$ antisera. To create an antigenic map we choose an embedding dimension, $d$, and then fit the $d(N+M-1)$ parameters specifying the locations of the $N$ antigens and $M$ sera (fixing one location at the 402 origin). The map thus created not only provides a visual interpretation of measured antigenic 403 distances, but also implicitly predicts unmeasured distances antigen/serum pairs. See S1 Text for 404 more information on our implementation of a maximum-likelihood based multidimensional 405 scaling algorithm for antigenic mapping.

406 HA1 genetic evolutionary model: Molecular evolution simulations are rooted on a mutational 407 algorithm that generates non-synonymous mutations in specific codons with a 2:1 transition to 408 transversion ratio, following Kimura's nucleotide evolution model [32]. In practice, a given 
number of mutations is assigned to a particular subset of amino acid positions and for each mutation up to 2 nucleotides from the respective codon are chosen to undergo mutation (keeping with a $2: 1$ the transition to transversion ratio). The procedure is repeated if necessary until one gets a non-synonymous substitution. This algorithm was implemented in Matlab® R2016a. We can thus generate hundreds of thousands of simulated viruses from any sampled H3 virus. We apply this DNA mutation model to 3 different genetic evolutionary frameworks expressing quite different mutation distribution profiles ranging from a model where mutations are restricted to only 7 amino acids [23]; to one where mutations are allocated to the 25 most significant amino acid positions as predicted by the RFA; and a generalized model where mutations are assigned to different amino acids according to a 4:1:2 ratio (RFA significant sites/other epitopes/other polymorphic sites).

\section{HA1 antigenic evolutionary model:}

Throughout this paper we try to ascertain the determinants of observed $\mathrm{H} 3$ antigenic evolutionary trends and consider the roles of physio-chemical plasticity ("plasticity"), immunological selection pressure ("antigenic filtering”), epidemiological seeding effects ("seeding").

The imposed physio-chemical plasticity is bounded by what is observed in the data, with mutations in simulated viruses having to comply to the observed minima and maxima in each amino acid position for the considered physio-chemical properties - S5 Figure.

Since the mean antigenic distance between viruses in two adjacent antigenic clusters is 4.46 $( \pm 1.3)$ on average), simulations complying to the "antigenic filtering" effect impose boundaries on simulated viruses' predicted antigenic distances to past clusters, according to two simple rules: 1) the simulated viruses must have predicted antigenic distance values within the 3-5 range 
relative to all viruses within the reference cluster; 2) predicted distances to viruses in all other past clusters must be greater than 6 . We thus discard any simulated viruses predicted to have an antigenic distance to viruses of the reference antigenic cluster greater than 5 or lower than 3 , and simultaneously a predicted antigenic distance lower than 6 to members of any other past cluster. We implemented the "seeding" effect as a 3-step mutational process (4-6 mutations in each step) where at the end of the $1^{\text {st }}$ and $2^{\text {nd }}$ steps one simulated virus is selected as a seed for the next simulation step.

Acknowledgments: We thank the EU FP7 EMPERIE project, the NIGMS MIDAS programme and the MRC for research funding.

\section{References:}

1. Nair H, Brooks WA, Katz M, Roca A, Berkley JA, Madhi SA, et al. Global burden of respiratory infections due to seasonal influenza in young children: a systematic review and meta-analysis. Lancet. 2011;378: 1917-1930. doi:10.1016/S0140-6736(11)61051-9

2. Bush RM. Predicting the Evolution of Human Influenza A. Science (80- ). 1999;286: 1921-1925. doi:10.1126/science.286.5446.1921

3. Smith DJ, Lapedes AS, de Jong JC, Bestebroer TM, Rimmelzwaan GF, Osterhaus ADME, et al. Mapping the antigenic and genetic evolution of influenza virus. Science. American Association for the Advancement of Science; 2004;305: 371-6. doi:10.1126/science.1097211

4. Sato K, Morishita T, Nobusawa E, Tonegawa K, Sakae K, Nakajima S, et al. Amino-acid change on the antigenic region B1 of $\mathrm{H} 3$ haemagglutinin may be a trigger for the emergence of drift strain of influenza A virus. Epidemiol Infect. 2004;132: 399-406. doi:10.1017/s0950268803001821

5. Raymond FL, Caton AJ, Cox NJ, Kendal AP, Brownlee GG. The antigenicity and evolution of influenza H1 haemagglutinin, from 1950-1957 and 1977-1983: Two pathways from one gene. Virology. 1986;148: 275-287. doi:10.1016/0042-6822(86)90325-9

6. Ferguson NM, Galvani AP, Bush RM. Ecological and immunological determinants of influenza evolution. Nature. 2003;422: 428-433. doi:10.1038/nature01509

7. Koelle K, Cobey S, Grenfell B, Pascual M. Epochal Evolution Shapes the Phylodynamics of Interpandemic Influenza A (H3N2) in Humans. Science (80- ). 2006;314: 1898-1903. doi:10.1126/science.1132745

8. Recker M, Pybus OG, Nee S, Gupta S. The generation of influenza outbreaks by a network of host immune responses against a limited set of antigenic types. Proc Natl Acad Sci. 2007;104: 77117716. doi:10.1073/pnas.0702154104

9. Koelle K, Rasmussen DA. The effects of a deleterious mutation load on patterns of influenza A/H3N2's antigenic evolution in humans. Elife. 2015;4: e07361. doi:10.7554/eLife.07361

10. Bedford T, Rambaut A, Pascual M. Canalization of the evolutionary trajectory of the human 
influenza virus. BMC Biol. BioMed Central; 2012;10: 38. doi:10.1186/1741-7007-10-38

11. Shih AC-C, Hsiao T-C, Ho M-S, Li W-H. Simultaneous amino acid substitutions at antigenic sites drive influenza A hemagglutinin evolution. Proc Natl Acad Sci. 2007;104: 6283-6288. doi:10.1073/pnas.0701396104

12. Du X, Dong L, Lan Y, Peng Y, Wu A, Zhang Y, et al. Mapping of H3N2 influenza antigenic evolution in China reveals a strategy for vaccine strain recommendation. Nat Commun. Nature Publishing Group; 2012;3: 709. doi:10.1038/ncomms1710

13. Luksza M, Lassig M. A predictive fitness model for influenza. Nature. 2014/02/28. 2014;507: 5761. doi:10.1038/nature13087nature13087 [pii]

14. Knossow M, Skehel JJ. Variation and infectivity neutralization in influenza. Immunology. 2006;119: 1-7. doi:10.1111/j.1365-2567.2006.02421.x

15. Skehel JJ, Wiley DC. Receptor Binding and Membrane Fusion in Virus Entry: The Influenza Hemagglutinin. Annu Rev Biochem. 2000;69: 531-569. doi:10.1146/annurev.biochem.69.1.531

16. Wilson IA, Cox NJ. Structural Basis of Immune Recognition of Influenza Virus Hemagglutinin. Annu Rev Immunol. 1990;8: 737-787. doi:10.1146/annurev.iy.08.040190.003513

17. Wiley DC, Wilson IA, Skehel JJ. Structural identification of the antibody-binding sites of Hong Kong influenza haemagglutinin and their involvement in antigenic variation. Nature. Nature Publishing Group; 1981;289: 373-378. doi:10.1038/289373a0

18. HIRST GK. THE AGGLUTINATION OF RED CELLS BY ALLANTOIC FLUID OF CHICK EMBRYOS INFECTED WITH INFLUENZA VIRUS. Science (80- ). 1941;94: 22-23. doi:10.1126/science.94.2427.22

19. Steinbrück L, McHardy AC. Allele dynamics plots for the study of evolutionary dynamics in viral populations. Nucleic Acids Res. 2011;39: e4-e4. doi:10.1093/nar/gkq909

20. Meyer AG, Wilke CO. Geometric Constraints Dominate the Antigenic Evolution of Influenza H3N2 Hemagglutinin. Bloom JD, editor. PLOS Pathog. Public Library of Science; 2015;11: e1004940. doi:10.1371/journal.ppat.1004940

21. Koel BF, Burke DF, Vliet S van der, Bestebroer TM, Rimmelzwaan GF, Osterhaus ADME, et al. Epistatic interactions can moderate the antigenic effect of substitutions in hemagglutinin of influenza H3N2 virus. bioRxiv. Cold Spring Harbor Laboratory; 2018; 506030. doi:10.1101/506030

22. Kryazhimskiy S, Dushoff J, Bazykin GA, Plotkin JB. Prevalence of Epistasis in the Evolution of Influenza A Surface Proteins. Malik HS, editor. PLoS Genet. 2011;7: e1001301. doi:10.1371/journal.pgen.1001301

23. Koel BF, Burke DF, Bestebroer TM, van der Vliet S, Zondag GC, Vervaet G, et al. Substitutions near the receptor binding site determine major antigenic change during influenza virus evolution. Science (80- ). 2013/11/23. 2013;342: 976-979. doi:10.1126/science.1244730342/6161/976 [pii]

24. Tria F, Pompei S, Loreto V. Dynamically correlated mutations drive human Influenza A evolution. Sci Rep. 2013;3: 2705. doi:10.1038/srep02705

25. Bahl J, Nelson MI, Chan KH, Chen R, Vijaykrishna D, Halpin RA, et al. Temporally structured metapopulation dynamics and persistence of influenza A H3N2 virus in humans. Proc Natl Acad Sci. 2011;108: 19359-19364. doi:10.1073/pnas.1109314108

26. Bedford T, Cobey S, Beerli P, Pascual M. Global Migration Dynamics Underlie Evolution and Persistence of Human Influenza A (H3N2). Ferguson NM, editor. PLoS Pathog. Public Library of Science; 2010;6: e1000918. doi:10.1371/journal.ppat.1000918

27. Russell CA, Jones TC, Barr IG, Cox NJ, Garten RJ, Gregory V, et al. The Global Circulation of Seasonal Influenza A (H3N2) Viruses. Science (80- ). American Association for the Advancement of Science; 2008;320: 340-346. doi:10.1126/SCIENCE.1154137

28. LAPEDES A, FARBER R. The Geometry of Shape Space: Application to Influenza. J Theor Biol. 2001;212: 57-69. doi:10.1006/jtbi.2001.2347

29. Hensley SE, Das SR, Bailey AL, Schmidt LM, Hickman HD, Jayaraman A, et al. Hemagglutinin receptor binding avidity drives influenza A virus antigenic drift. Science (80- ). 2009/11/11. 
30. Breiman L. Random Forests. Mach Learn. Kluwer Academic Publishers; 2001;45: 5-32. doi:10.1023/A:1010933404324

31. Díaz-Uriarte R, Alvarez de Andrés S. Gene selection and classification of microarray data using random forest. BMC Bioinformatics. BioMed Central; 2006;7: 3. doi:10.1186/1471-2105-7-3

32. Kimura M. A simple method for estimating evolutionary rates of base substitutions through comparative studies of nucleotide sequences. J Mol Evol. Springer-Verlag; 1980;16: 111-120. doi:10.1007/BF01731581 paper.

a

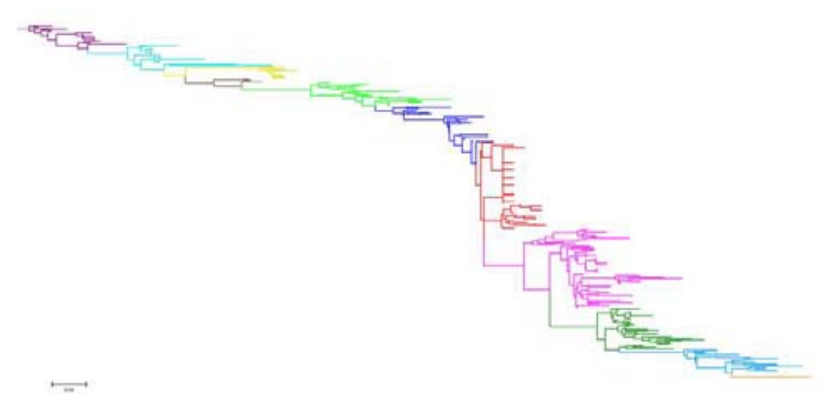

b
C
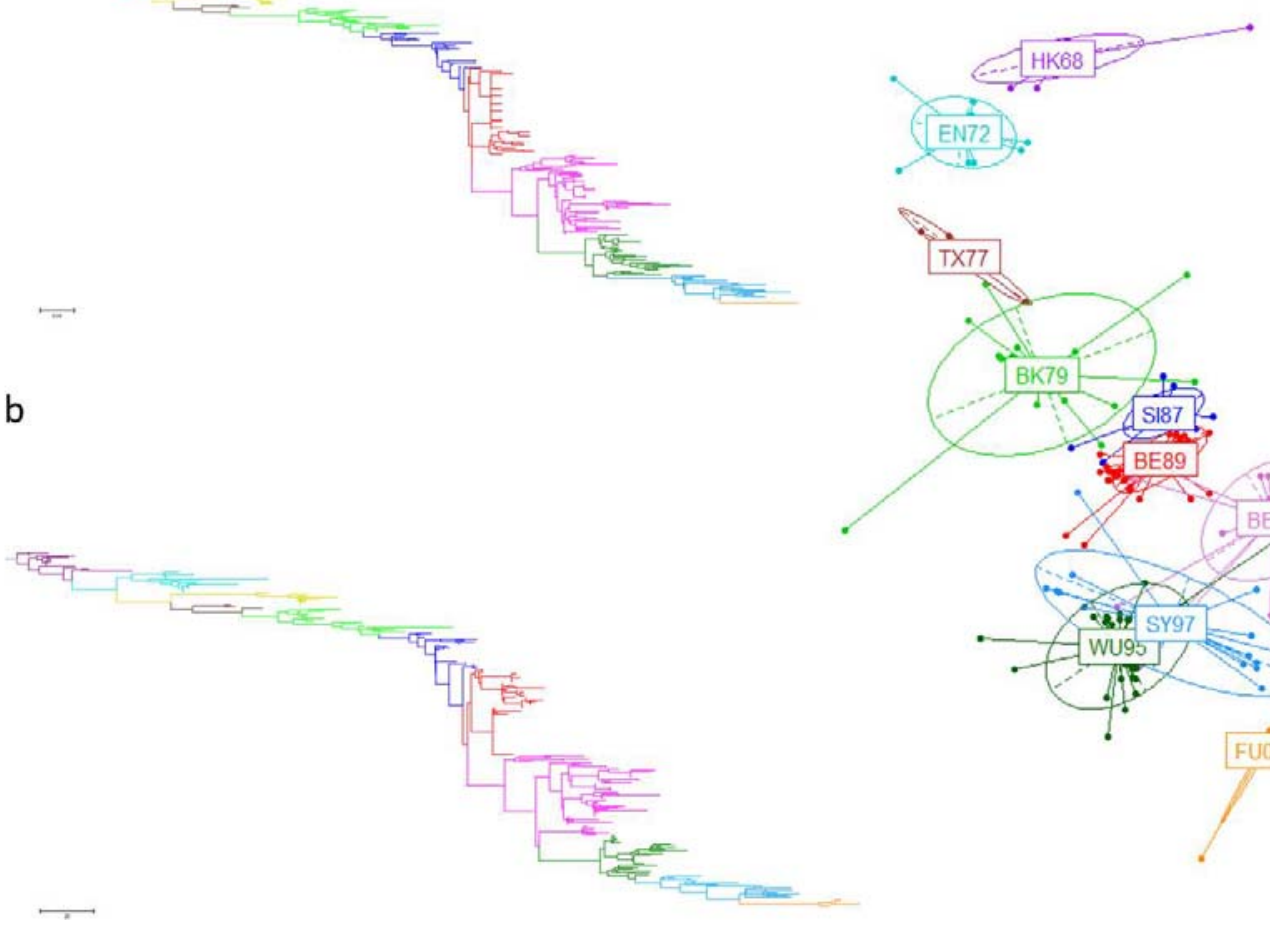

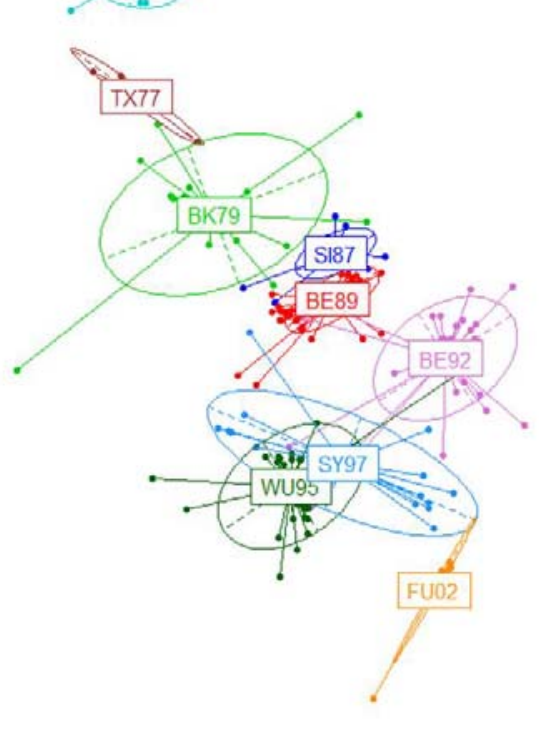


536 S1 Figure. Correspondence between genetic distances and sum in polarity and

537 hydrophobicity across the sequences. (A) ML phylogenetic tree of all antigen sequences. (B)

538 Dendrogram built from a distance matrix derived from the sum of differences in polarity and

539 hydrophobicity in the antigen sequences. (C) MDS using the same matrix as in (B).

540 
a

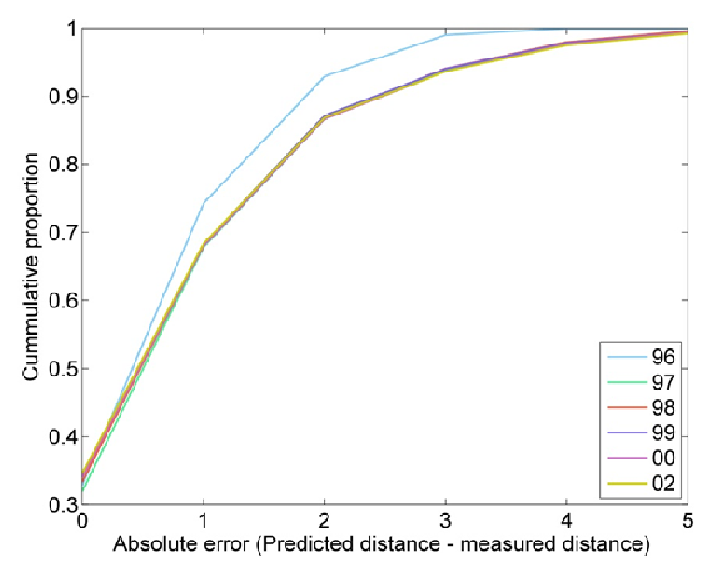

b

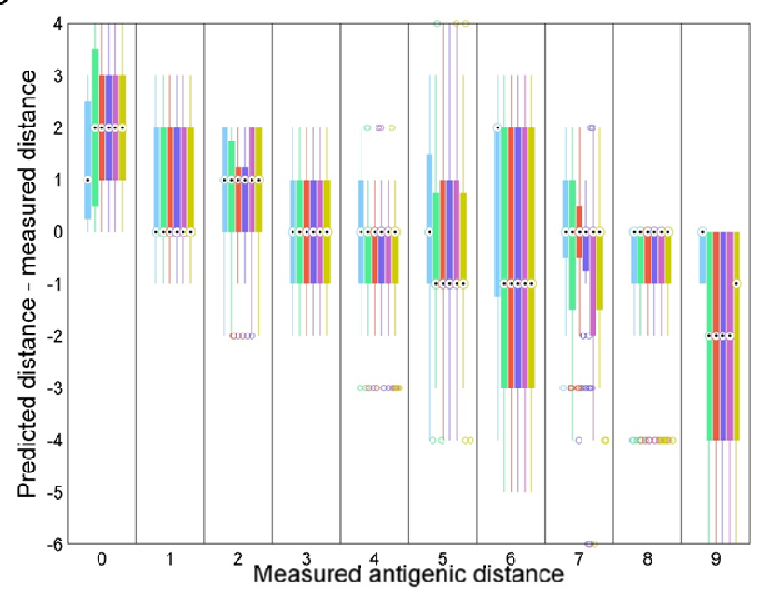

C
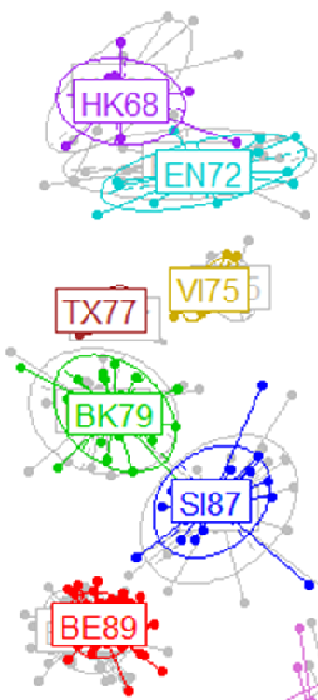

BE92

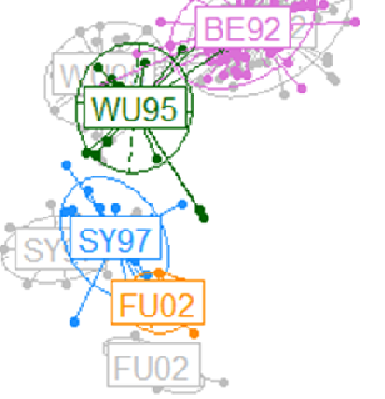

544 distribution of the prediction errors (on a $\log -2$ scale) for the different prediction time windows.

545 (B) Box plots of the prediction error for specific values of measured antigenic distance. For each

546 box, the central mark represents the median, the edges mark the $25^{\text {th }}$ and $75^{\text {th }}$ percentiles, and the

547 whiskers extend to the most extreme data points not considered outliers. Here the whiskers

548 extend to $+/-2.7 *$ variance (corresponding to 99.3 coverage assuming the data are normally

549 distributed). Each colour represents an incremental prediction time window as in (A). (C) 
bioRxiv preprint doi: https://doi.org/10.1101/770446; this version posted September 24, 2019. The copyright holder for this preprint (which was not certified by peer review) is the author/funder. All rights reserved. No reuse allowed without permission.

550 Predicted antigenic map with predictions in colour overlaid on the map generated with full 551 hindsight (in grey). 


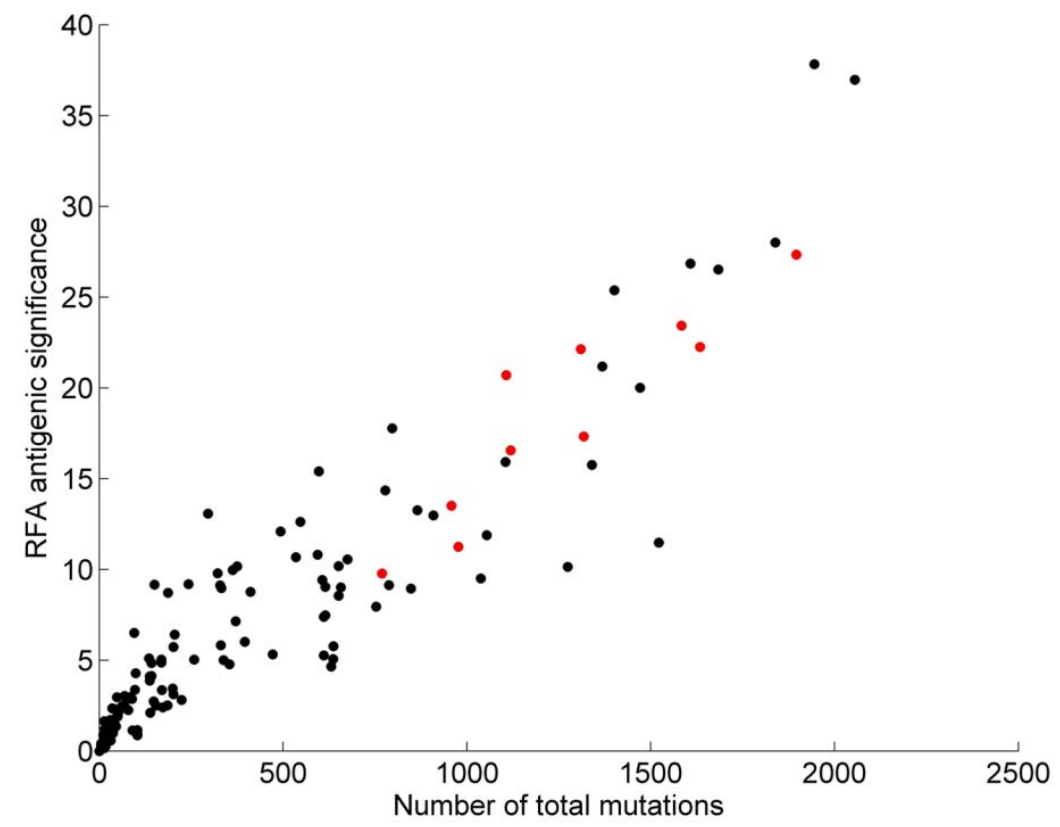

553 S3 Figure. Correlation between the total number of mutations recorded at each position

554 and the respective RFA (trained on the full dataset) significance. Amino acid positions with

555 high interaction indices are highlighted in red. 

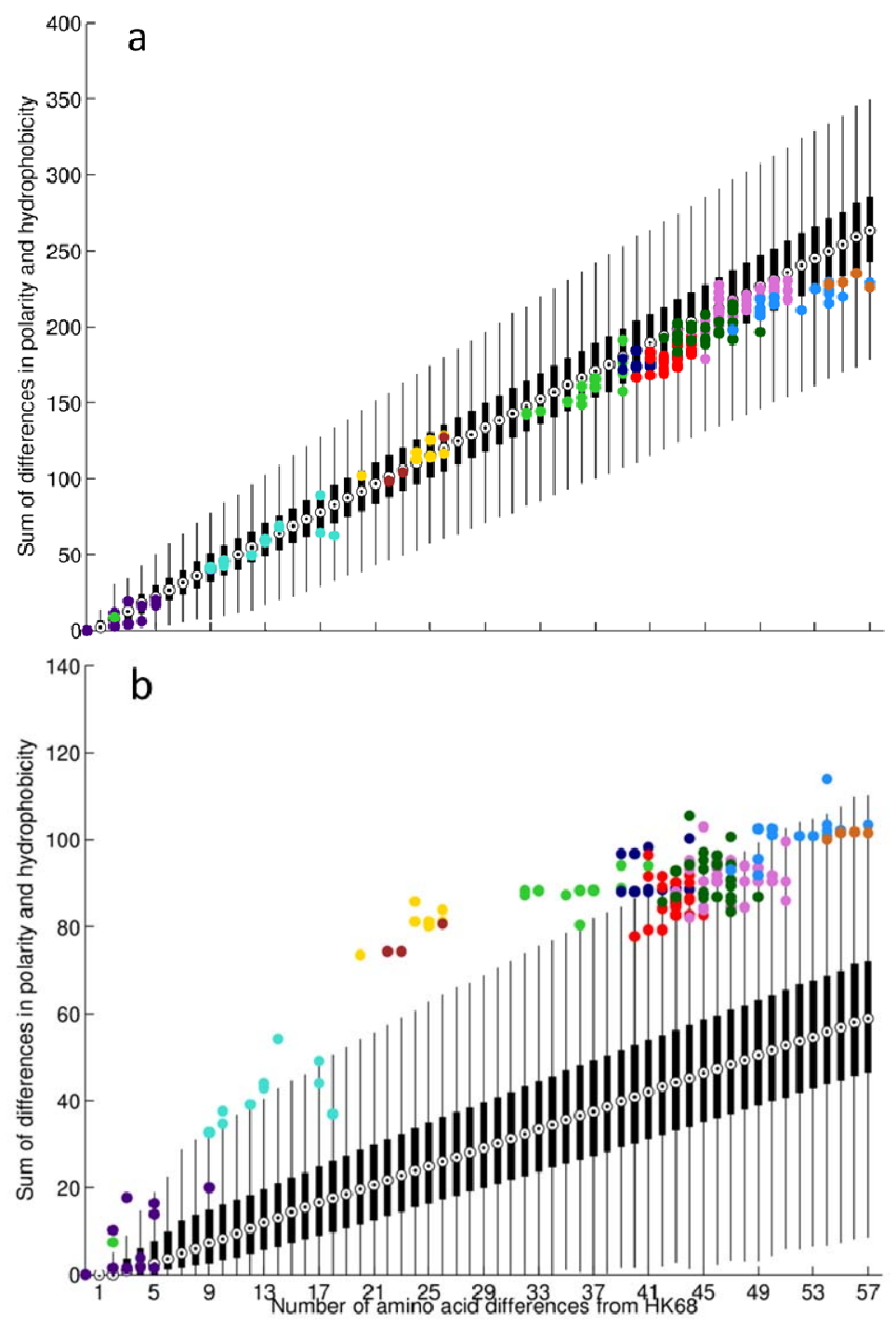

S4 Figure. Evolution in HA1. Each box plot in black show the variation in the sum of 559 differences in amino-acid polarity and hydrophobicity between 100,000 viruses and their 560 ancestral HK68 virus (given a specific number of mutations). The black circle in each box 561 identifies the median, the edges mark the 25 th and 75 th percentiles, and the whiskers extend to 562 the most extreme data points not considered outliers. (A) Shows differences across the full HA1 563 sequence, and (B) across epitope positions only. Isolated viruses are color-coded according to 564 their assigned antigenic cluster. 

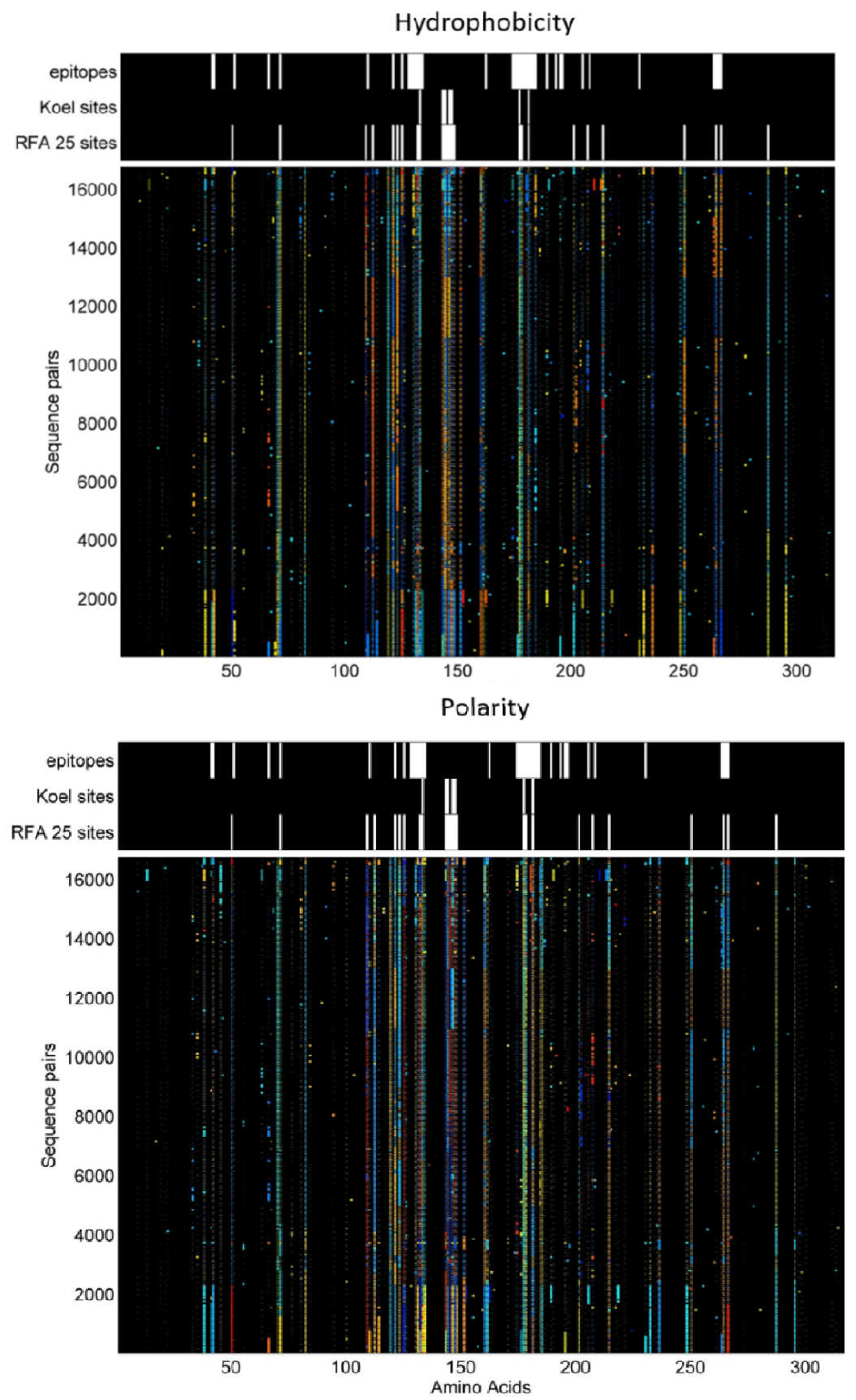

567 S5 Figure. HA1 physiochemical plasticity. Displays the observed difference in hydrophobicity

568 (top) and polarity (bottom) for all antigen/serum pairs (rows) in each amino acid position

569 (columns). Black indicates no difference, whereas blue and red represent the most extreme

570 values (negative and positive, respectively). 


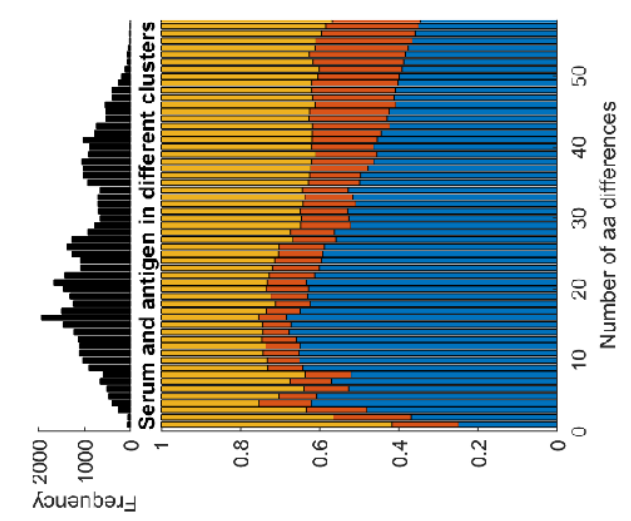

572 S6 Figure. HA1 mutational profile. Shows the distribution of mutations across three subsets of

573 amino acids. Blue illustrates mutations that fall on the subset of RFA 25 most significant sites, in

574 red we represent the known epitopes which are not included in the blue subset, and in orange the

575 remaining polymorphic sites (not included in any of the other subsets). 


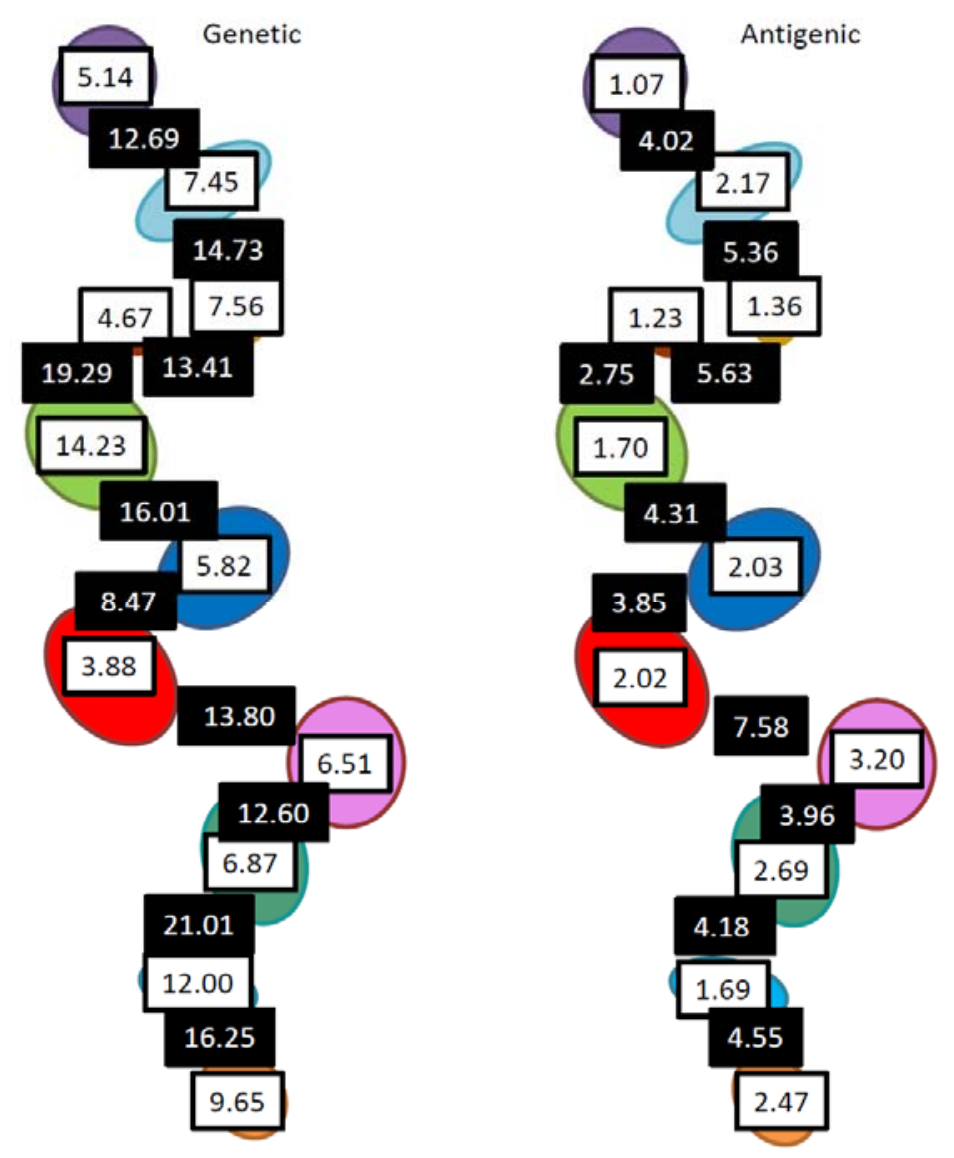

578 S7 Figure. H3 Antigenic and Genetic Evolutionary patterns. This sketch illustrates mean

579 differences between members of the same cluster (white background boxes) and between viruses

580 belonging to contiguous clusters (black background). The mean antigenic differences shown here

581 are calculated from the HI titers measured in the considered antigen/sera pairs. The mean genetic

582 differences are merely the mean number of amino acid differences between sequences of the

583 same cluster or across contiguous clusters. 
a
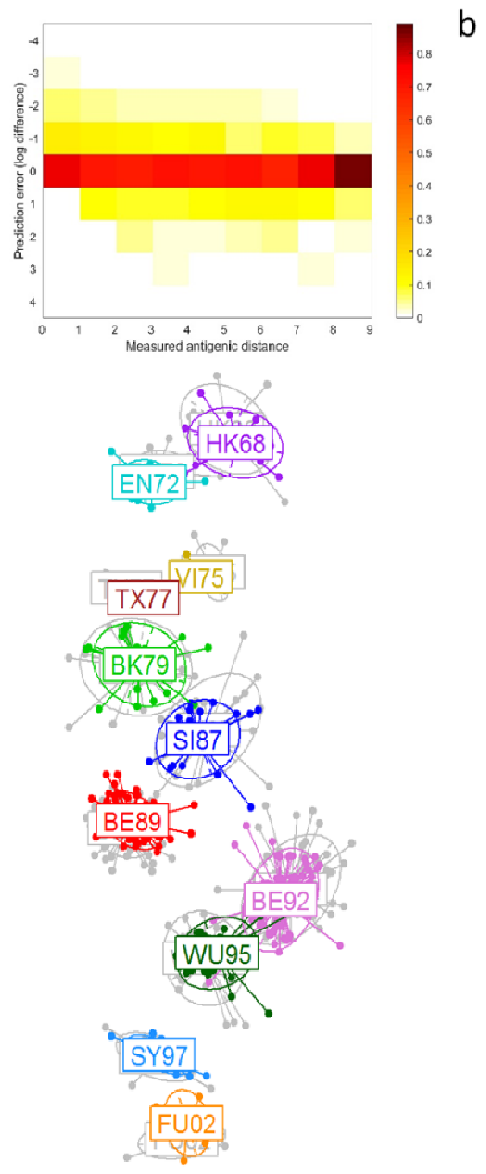
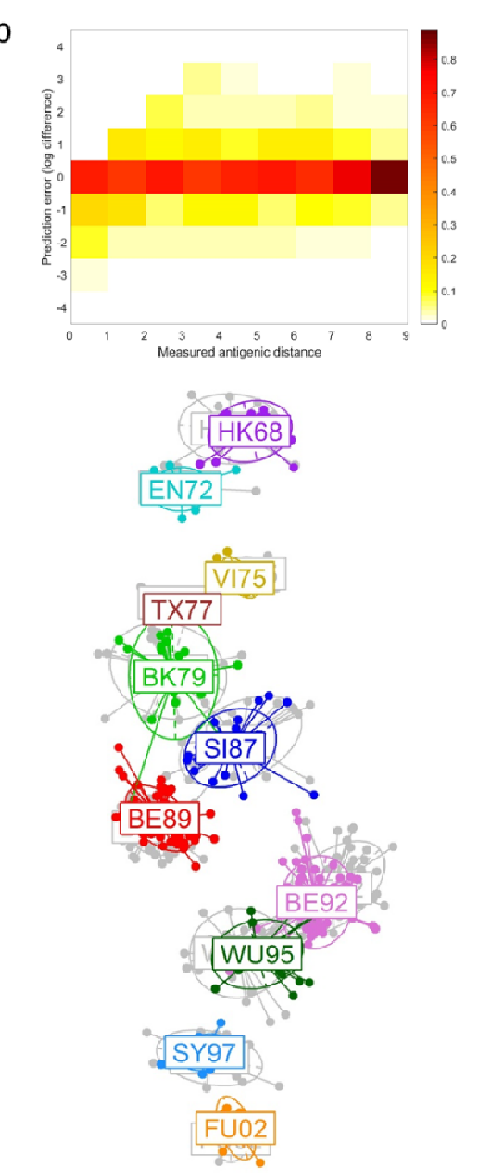
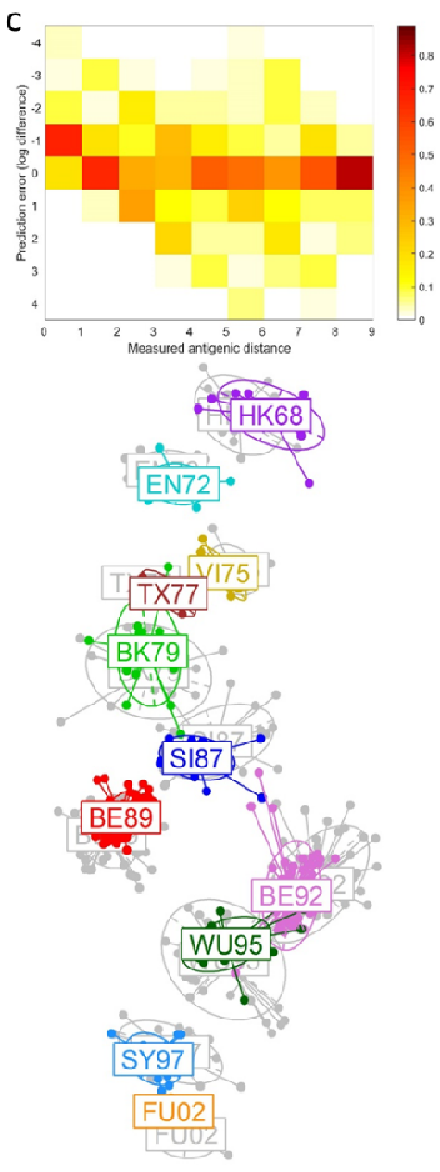

S8 Figure. Evolutionary model accuracy. Classification accuracy of the RFA looking at

589 hydrophobicity and polarity differences in each of the considered evolutionary models: the 590 generalized (A); the RFA 25 most significant sites (B) or the 7 sites model proposed by Koel et

591 al (C). Superimposed 2-dimensional antigenic maps generated from the predicted antigenic distances (in colour) - when training the RFA on the respective subsets of amino acids - and from the HI titre data (in grey). The top panels give the prediction errors for each of the observed antigenic distance bins. 
a
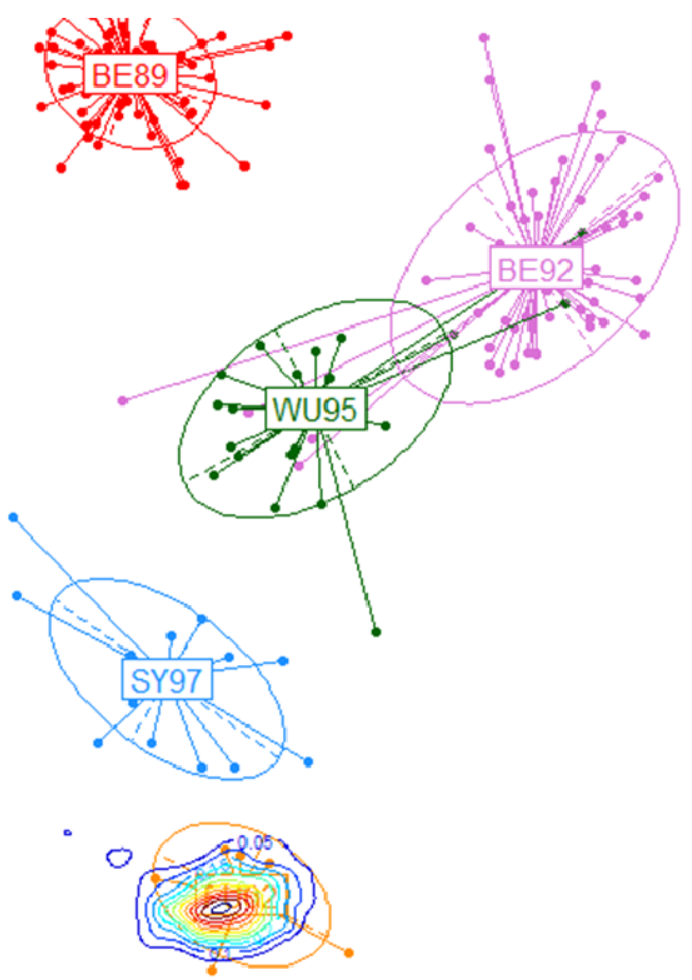

b

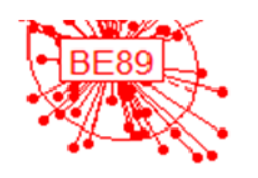

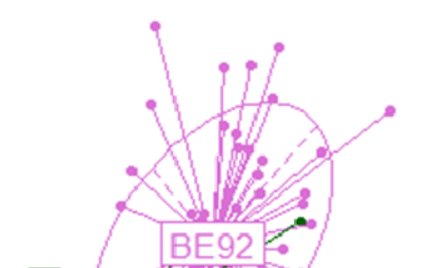

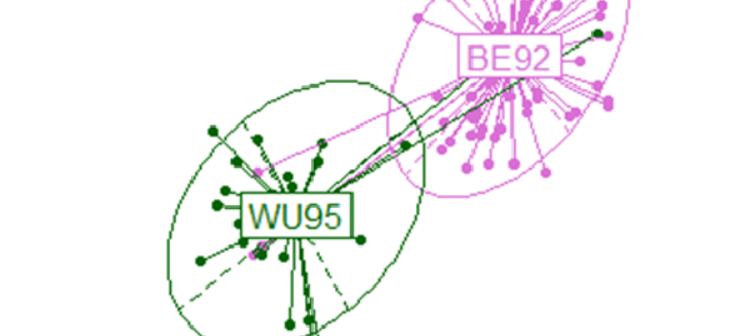

596

597 S9 Figure. Antigenic maps of influenza's predicted antigenic trajectories. The contour lines

598 represent the density probability that simulated viruses will fall on the respective delineated 599 antigenic space. The simulated viruses were generated from mutations in: (A) significant 600 positions to explain antigenic differences between clusters BE92 to FU02; (B) the polymorphic 601 sites across the SY97and FU02 clusters. Each simulated virus has a total of 12-18 amino acid 602 mutations relative to its ancestor.

603 
a
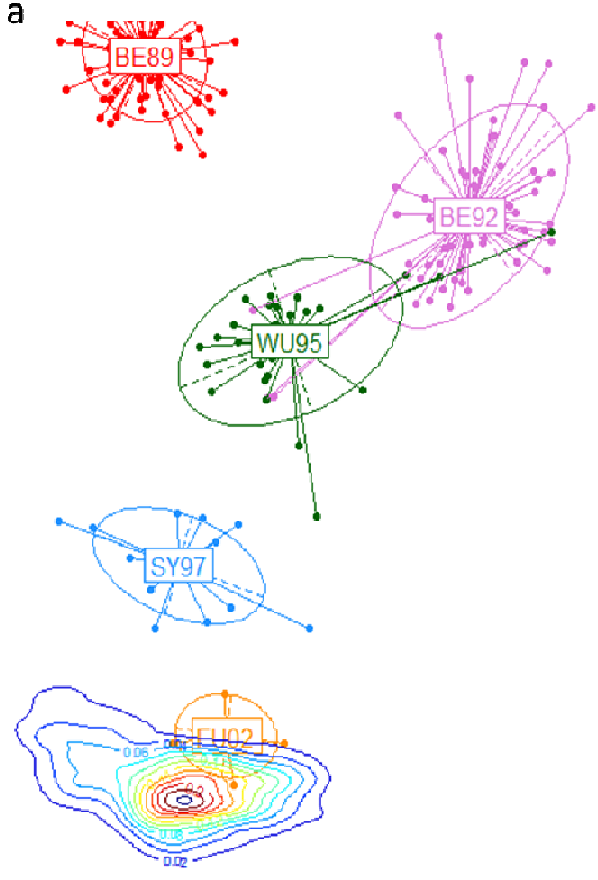

b

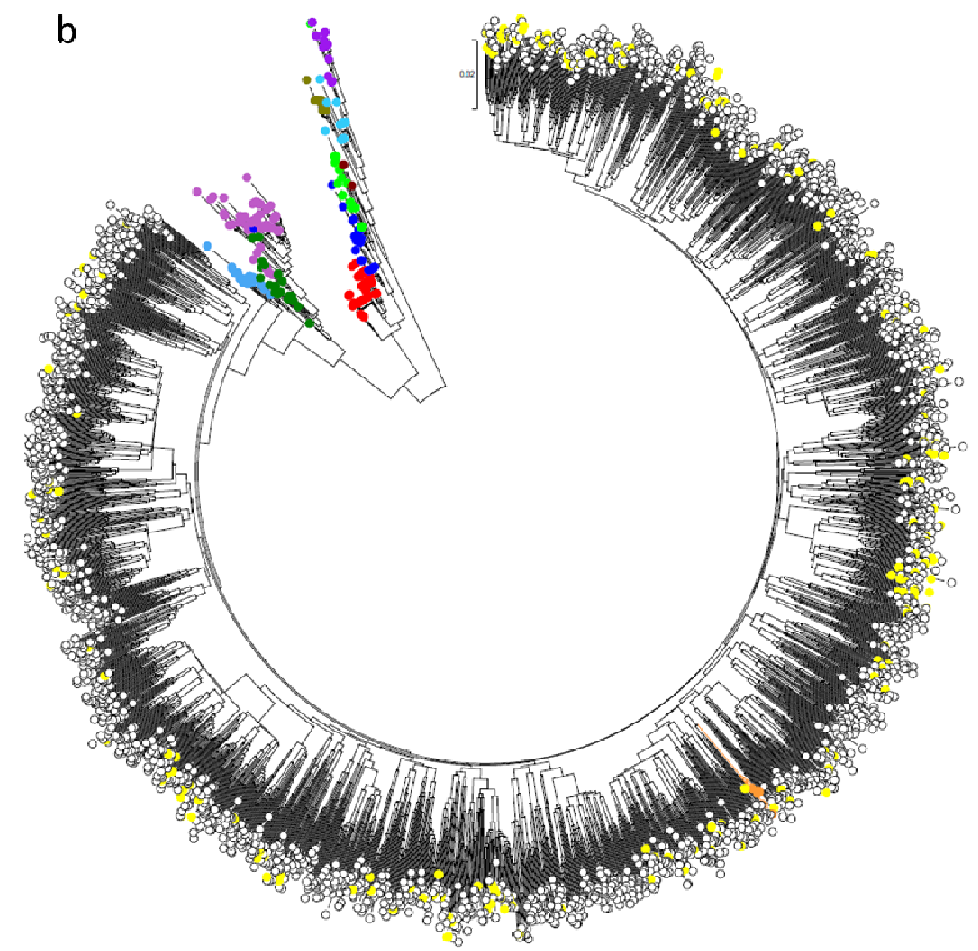

605

606

607

608

609

610

611

612

613

614

615 simulated viruses.

S10 Figure. Antigenic trajectory unpredictability. Antigenic map of influenza's predicted antigenic trajectory using the generalized model (A). We implemented a mutational process similar to that in S9 Figure and impose an antigenic filter that only accepts simulated viruses with predicted antigenic distances to the ancestral viral cluster in $[3,5]$ and of over 6 to all preceding clusters. The contour lines represent the density probability that simulated viruses will fall on the respective delineated antigenic space. A maximum likelihood neighbor joining tree shows the genetic relationship between simulated viruses that did not meet the antigenic filter requirements (white), those that did (yellow), and the empirical viruses (colored according to 

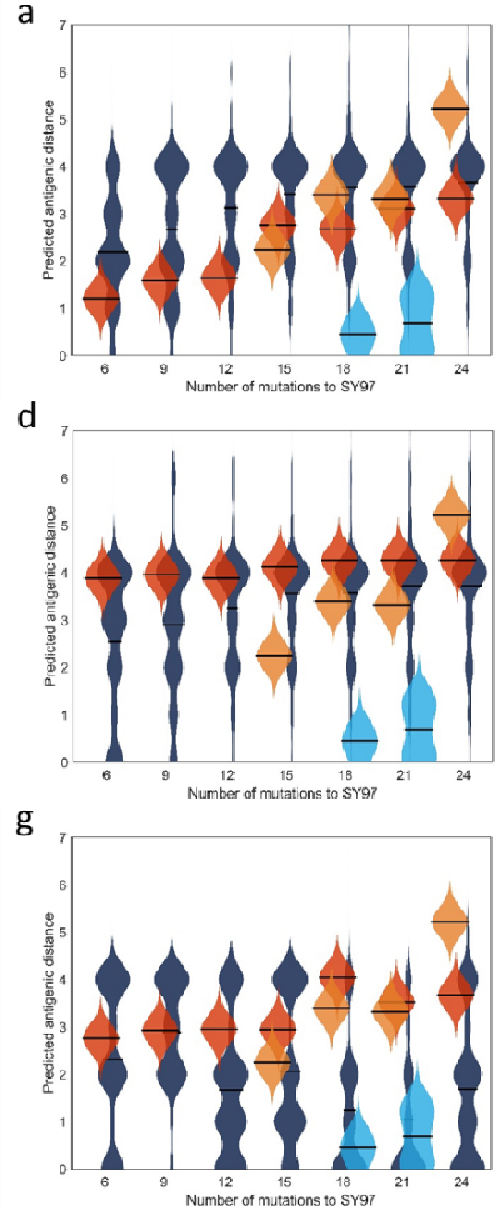

b

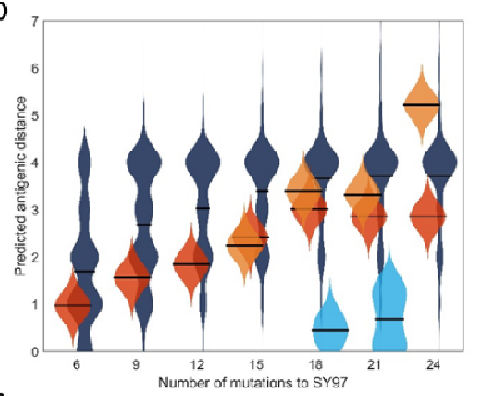

e

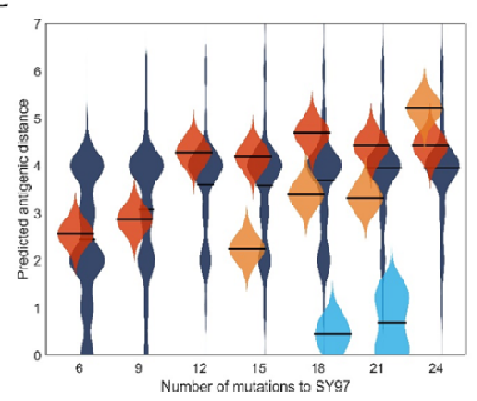

h

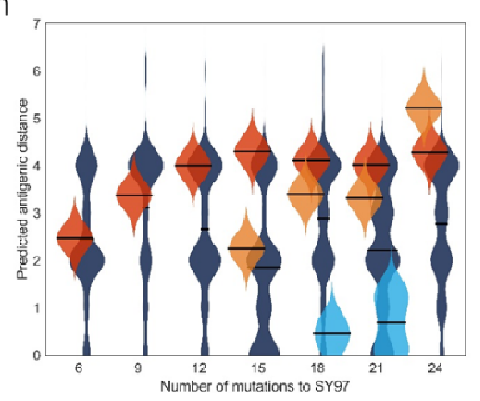

C

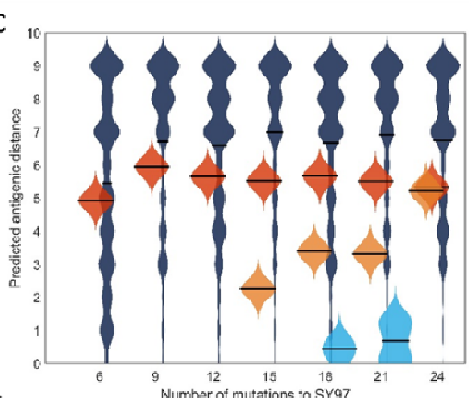

$f$

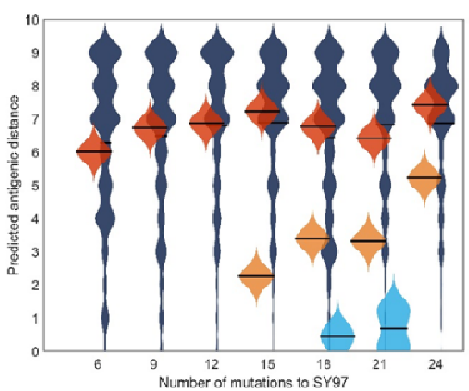

i

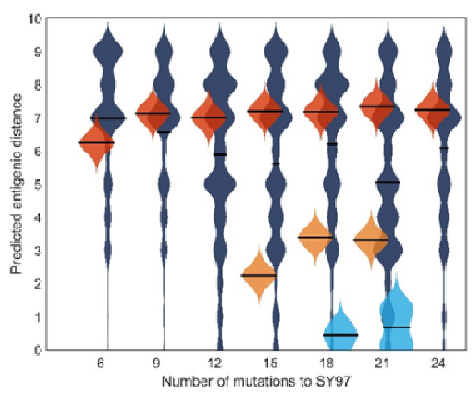

S11 Figure. Antigenic trajectories and underlying evolutionary processes. Unfiltered (top row); filtered (middle row); and filtered step-wise (bottom row) antigenic evolution from a specific ancestral strain under the 3 evolutionary models. The results for the generalized, 25-site, and 7-site models are depicted left to right respectively. The dark blue violin plots represent the distribution of antigenic distances (with the respective means as horizontal lines) between simulated viruses with $\mathrm{X}$ amino acid mutations relative to a virus in SY97. The red violin plots give the predicted antigenic distances across simulated viruses. Light blue violin plots give the empirical antigenic distances between viruses in SY97 and FU02, whilst the orange plots refer to the measured antigenic distances within the FU02 cluster. 


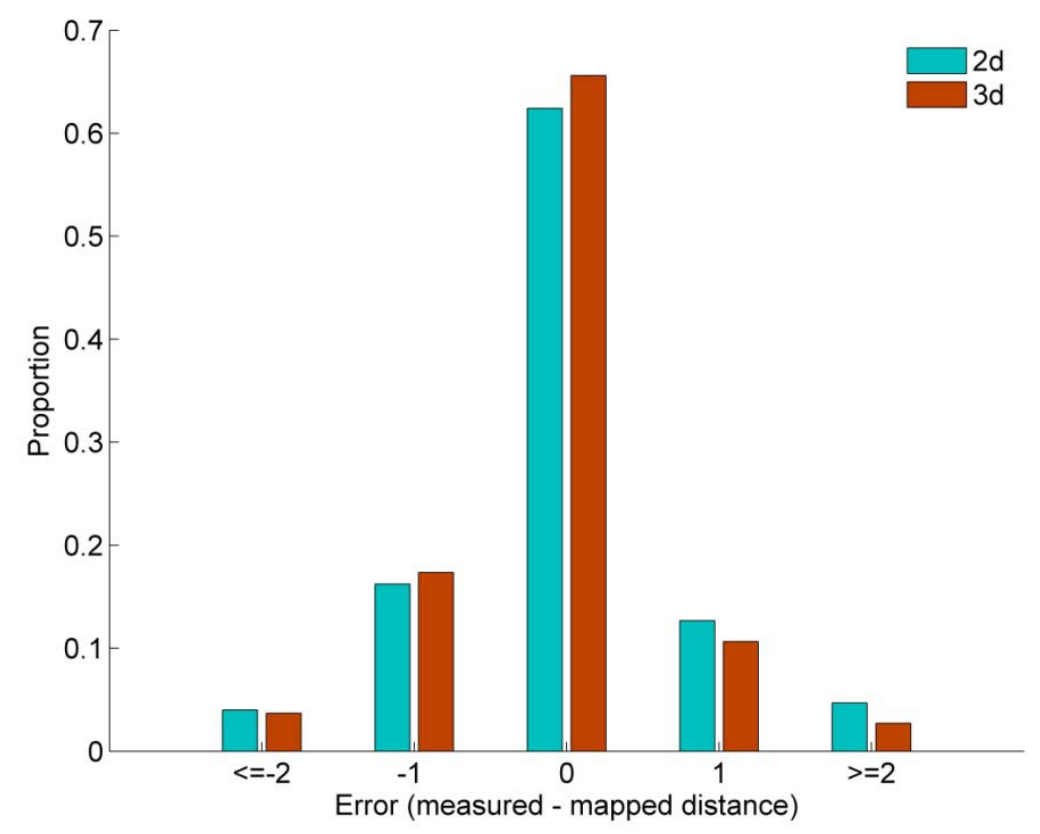

630 S12 Figure. Antigenic map precision on different embeddings. Comparison of the MDS error

631 for the 2D and 3D representations of an antigenic map with two simulated lineages.

632

633

634 
636 S13 Figure. Genetic determinants of simulated viral lineages. Each panel relates to the

637 associated cluster jump in Figure 5 of the main text. The left plot of each panel shows the DAPC

638 resulting discrimination of the separate groups given the genetic information. The contribution of

639 each allele for the segregation of the viruses into groups is shown on the plots to the right. The

640 dashed line serves only as an arbitrary threshold above which the individual allele labels are 641 shown. 


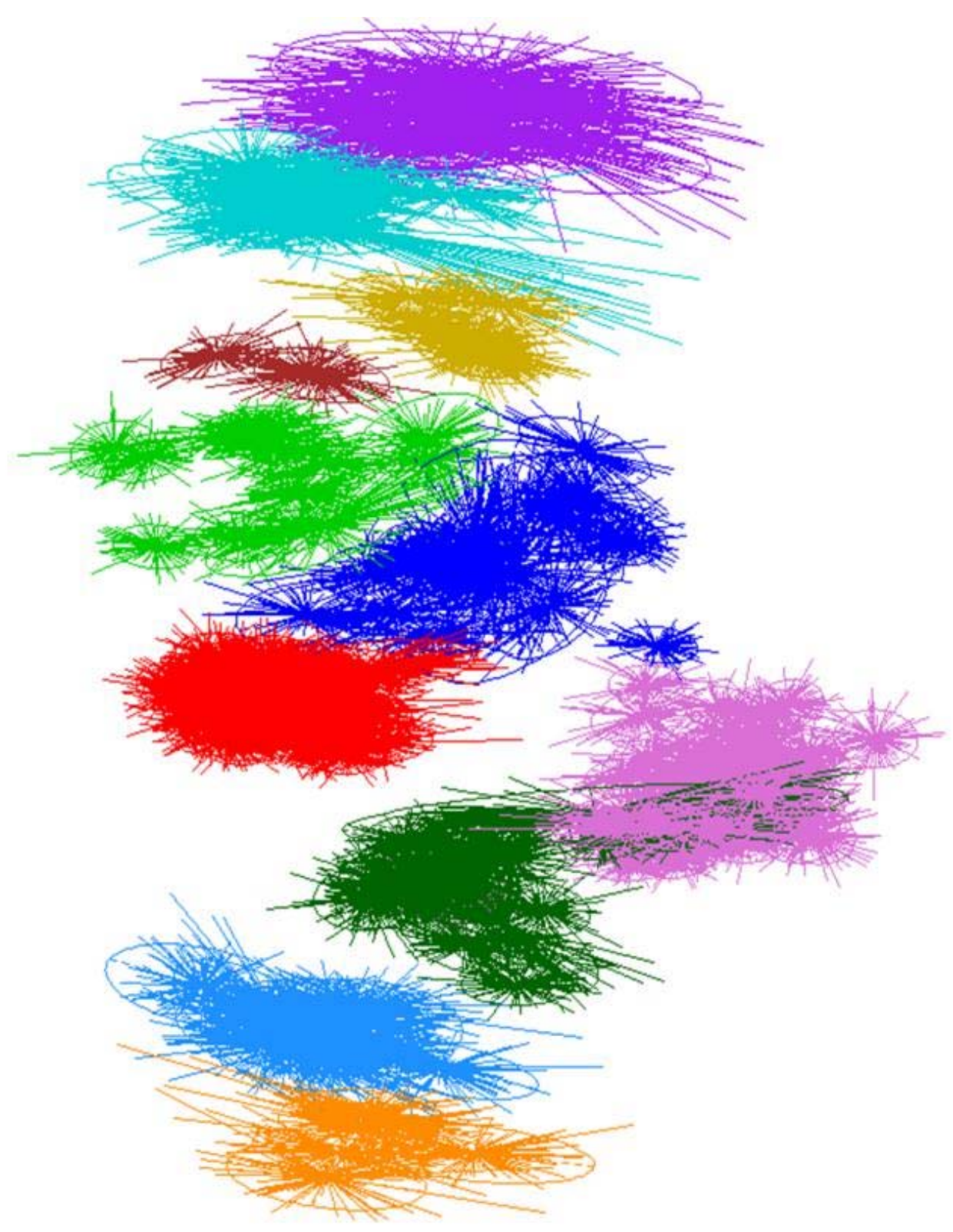

644 S14 Figure. Uncertainty of the antigenic mapping algorithm of historic H3 influenza. Each

645 ellipsis represents the dispersal in the final proposed coordinates for each antigen (over 100

646 bootstrap runs of the mapping algorithm). Colors match the cluster assignments in . 
a

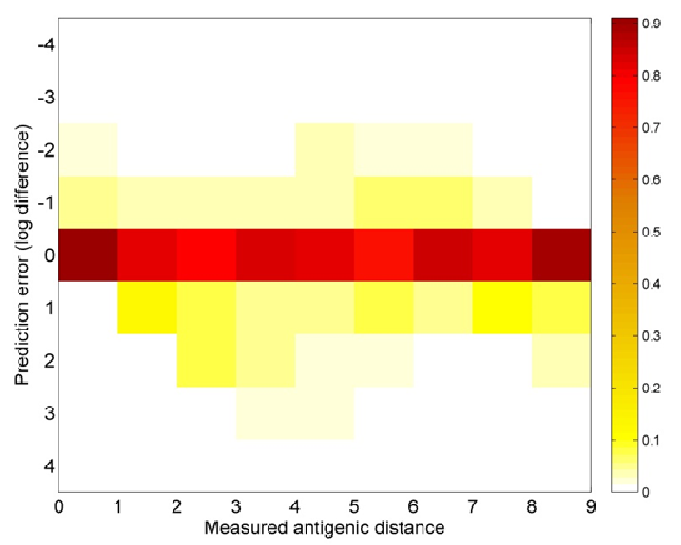

b

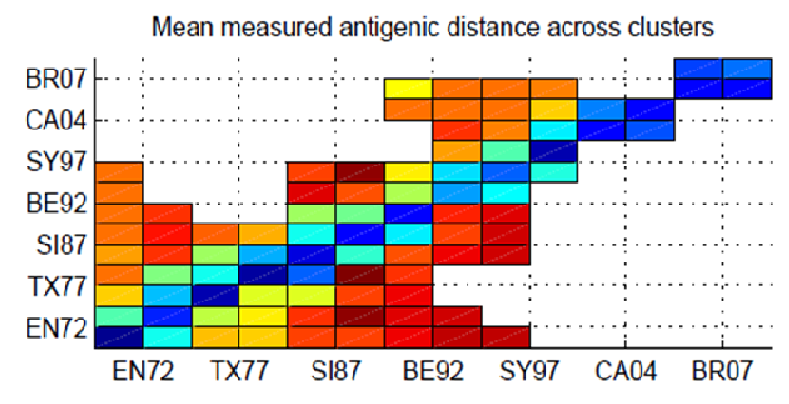

C

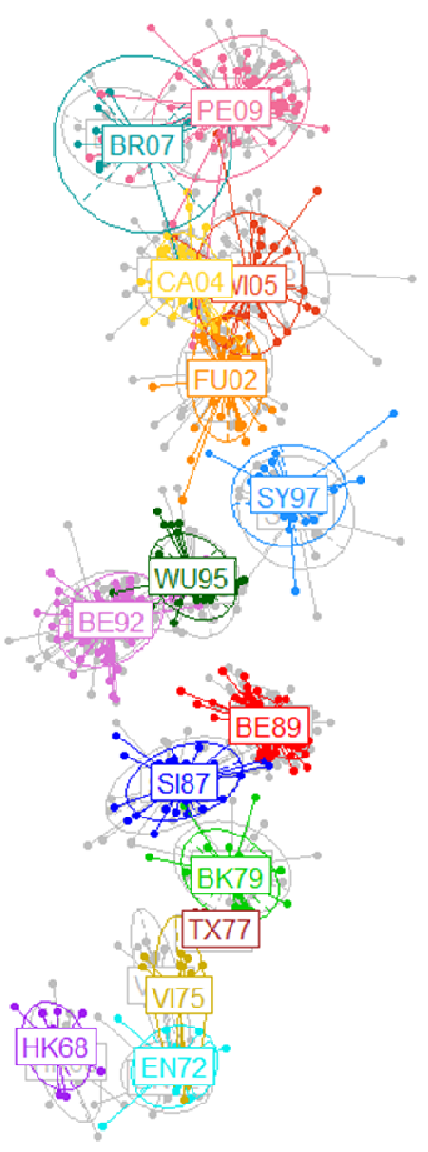

648

649

650 S15 Figure. Classification accuracy of the RFA using hydrophobicity and polarity as

651 antigenic proxy variables for an extended dataset. (A) Surface plot showing the error in

652 prediction of antigenic distance (distance being measured on a log-2 scale) for every antigen-

653 serum pair. Antigen-serum pairs are grouped by measured antigenic distance on the horizontal

654 axis. The colour of each rectangular pixel shows the proportion of pairs with at a particular

655 measured antigenic distance with a particular prediction error. (B) Mean measure antigenic

656 distance between antigen/serum pairs composed of elements of different antigenic clusters. The

657 sparseness of this matrix of measurements increases significantly for more recent data. (C) 
658 Superimposed 2-dimensional antigenic maps generated from the RFA predicted antigenic

659 distances (in colour) and from the HI titre data (in grey).

660

661

662

663

664

665 